\title{
Rare-event Simulation and Efficient Discretization for the Supremum of Gaussian Random Fields
}

\author{
Xiaoou Li and Jingchen Liu \\ Columbia University
}

September 17, 2018

\begin{abstract}
In this paper, we consider a classic problem concerning the high excursion probabilities of a Gaussian random field $f$ living on a compact set $T$. We develop efficient computational methods for the tail probabilities $P\left(\sup _{T} f(t)>b\right)$ and the conditional expectations $E\left(\Gamma(f) \mid \sup _{T} f(t)>\right.$ $b)$ as $b \rightarrow \infty$. For each $\varepsilon$ positive, we present Monte Carlo algorithms that run in constant time and compute the interesting quantities with $\varepsilon$ relative error for arbitrarily large $b$. The efficiency results are applicable to a large class of Hölder continuous Gaussian random fields. Besides computations, the proposed change of measure and its analysis techniques have several theoretical and practical indications in the asymptotic analysis of extremes of Gaussian random fields.
\end{abstract}

\section{Introduction}

In this paper, we consider the design and the analysis of efficient Monte Carlo methods for the high excursion events of Gaussian random fields. Consider a probability space $(\Omega, \mathcal{F}, P)$ and a Gaussian random field

$$
f: T \times \Omega \rightarrow R
$$

living on a $d$-dimensional compact subset $T \subset R^{d}$. Most of the time, we omit the second argument and write $f(t)$. Let $M=\sup _{t \in T} f(t)$. In this paper, we are interested in the efficient computation of the high excursion probabilities of $f(t)$, that is,

$$
w(b) \triangleq P(M>b)
$$

and the corresponding conditional expectations

$$
v(b) \triangleq E(\Gamma(f) \mid M>b)
$$

in the asymptotic regime that $b$ tends to infinity, where $\Gamma(\cdot)$ is a functional (possibly a random functional) mapping from the space of continuous functions to the real line.

The proposed algorithms are based on importance sampling that is associated with an appropriately designed change of measure mimicking the conditional distribution $P(f \in \cdot \mid M>b)$. Much of this paper will focus on the design and the implementation of the algorithm for the tail probability

$w(b)$. For the conditional expectation, we present an efficient algorithm and its analysis for one specific example: the integral on the excursion set with respect to positive processes. It turns out that the computations of $w(b)$ and $v(b)$ are closely related, which will be discussed in details in 
Section 2.2. Most of the time, we are interested in computing small quantities converging to zero. Thus, it is sensible to consider relative accuracy that is defined as follows.

Definition 1 For some positive $\varepsilon$ and $\delta$, a Monte Carlo estimator $Z$ of $w$ is said to admit $\varepsilon-\delta$ relative accuracy if

$$
P(|Z-w|<\varepsilon w)>1-\delta .
$$

We propose Monte Carlo estimators admitting $\varepsilon-\delta$ relative accuracy for computing the tail probabilities $w(b)$ and the conditional expectations $v(b)$. One notable feature of this estimator is that the total computational complexity to generate one such estimator is bounded by a constant $C(\varepsilon, \delta)$ that is independent of the excursion level $b$. Thus, to compute $w(b)$ and $v(b)$ with any prescribed relative accuracy as in (3), the total computational complexity remains bounded as the event becomes arbitrarily rare. With such an algorithm, the computation of rare event is at the same level of complexity as the computation of regular expectations. In addition, this efficiency result is applicable to a large class of Hölder continuous Gaussian random fields and thus is very generally applicable.

The analysis mainly consists of two components. First, we propose a change of measure on the continuous sample path space (denoted by $Q_{b}$ ). The corresponding importance sampling estimators are unbiased. The first step of the analysis is to show that the estimators admit standard deviations on the order of $O(w(b))$ or $O(v(b))$. Such estimators are said to be strongly efficient that is a common efficiency concept in the rare-event simulation literature ([13, 5]).

The second part of the analysis concerns the implementations. The simulation of the estimators in the previous paragraph requires the generation of the entire sample path of $f$. Under the current context, the process $f$ is a continuous function. Computer can only perform discrete simulations. Therefore, we need to seek for an appropriate discretization scheme to perform the simulations. For instance, a natural approach is to choose a subset

$$
T_{m}=\left(t_{1}, \ldots, t_{m}\right) \subset T
$$

and use the discrete field living on $T_{m}$ to approximate the continuous field. Thanks to continuity and under certain regularity conditions of $T_{m}$, one can show that $P\left(\sup _{T_{m}} f(t)>b\right) / w(b) \rightarrow 1$ as $m \rightarrow \infty$, i.e., the bias vanishes as the size of the discretization increases. However, it is well understood that this convergence is not uniform in $b$. The smaller $w(b)$ is, the slower it converges, indicating that the set $T_{m}$ needs to grow in order to maintain a prefixed relative bias. In fact, as discussed in [2, for any deterministic subset $T_{m}$, the size $m$ must increase at least polynomially with $b$ to ensure a given relative accuracy. In this paper, the discretization scheme is random and adapted to (correlated with) the random field $f$. This adaptive scheme substantially reduces the computation complexity, in particular, to a constant level.

The high level excursion of Gaussian random fields is a classic topic in probability. There is a wealth of literature that contains general bounds on $P(\sup f(t)>b)$ as well as sharp asymptotic approximations as $b \rightarrow \infty$. A partial literature contains [17, 22, 25, 10, 12, 18, 27, 8]. Several methods have been introduced to obtain bounds and asymptotic approximations, each of which imposes different regularity conditions on the random fields. General upper bound for the tail of $\max f(t)$ is developed in [10, 31], which is known as the Borel-TIS lemma. For asymptotic results, there are several methods. The double sum method (24]) requires an expansion of the covariance function around its global maximum and also locally stationary structure. The Euler-Poincaré Characteristics of the excursion set approximation (denoted by $\chi\left(A_{b}\right)$, where $A_{b}$ is the excursion set) uses the fact $P(M>b) \approx E\left(\chi\left(A_{b}\right)\right)$ and requires the random field to be at least twice differentiable $([1,28,4,29])$. The tube method ([26]) uses the Karhunen-Loève expansion and imposes 
differentiability assumptions on the covariance function (fast decaying eigenvalues) and regularity conditions on the random field. The Rice method $([6,7])$ represents the distribution of $M$ (density function) in an implicit form. For other convex functionals, the exact tail approximation of integrals of exponential functions of Gaussian random fields is developed by [19, 21]. Recently, [3] studied the geometric properties of high level excursion set for infinitely divisible non-Gaussian fields as well as the conditional distributions of such properties given the high excursion. Numerical methods are recently discussed by [2] who proposes importance sampling estimators of $w(b)$. In particular, the authors show that the proposed estimator is a fully polynomial randomized approximation scheme (FPRAS), that is, to achieve the $\varepsilon-\delta$ relative accuracy, the total computation complexity is of order $O\left(\varepsilon^{-q_{1}} \delta^{-q_{2}}|\log w(b)|^{q}\right)([30,33,23])$. When $w(b)$ is very small, the complexity $O\left(|\log w(b)|^{q}\right)$ could be computationally heavy.

This paper is a nontrivial and substantial generalization of [2]. In particular, the contributions are as follows. First, we introduce an adaptive discretization scheme that reduces the overall computational cost to a constant level. This is a substantial improvement of [2] who requires the discretization size grow polynomially in $b$ for both differentiable and non-differentiable fields. Second, we show that the continuous importance sampling estimator is strongly efficient to compute $w(b)$ for both Hölder continuous fields and differentiable cases (by imposing mild regularity conditions). This generalizes the results in [2] who establishes that their relative error grows polynomially fast with $b$ unless the process is twice differentiable for which the exact Slepian model is available. Third, we present an algorithm with constant complexity for the computation of the conditional expectations of integrals on the excursion sets. Lastly, from the technical and methodological point of view, the development of this paper mostly deals with change of measures defined on the continuous sample path space. In contrast, the analysis of [2] relies heavily on the discrete nature of the estimators (multivariate Gaussian random vectors). The methodological contribution of this paper is developing techniques to deal with change of measures defined on the continuous sample path space. As we shall see in the technical development, with moderate adaptations, the analysis techniques can be applied to the analysis of a large class of conditional expectations $E[\Gamma(f) \mid M>b]$. In particular, in Theorem 3 , we employ the change of measure to derive the asymptotic approximations of the expected conditional integrals.

As the total complexity of the Monte Carlo estimator is constant, the computational cost is comparable to that of the closed form approximation of $w(b)$. One advantage of our method is that it can yield arbitrarily small relative error at the expense of more computational costs; while the error of closed form approximations are prefixed for each $b$ and they are usually not straightforward to obtain - requiring second order approximations. In addition to $w(b)$, the current Monte Carlo methods can also be used to compute the conditional expectations whose asymptotic analyses are case-by-case. Another advantage of the Monte Carlo estimators is that their implementations do not require the computation of various constants appearing in the closed form approximations (such as Pickands constant, Lipschitz-Killing Curvature, etc), neither do they require the fine knowledge of the local expansions. In addition to the tail probabilities and the conditional expectations, the proposed estimator also provides means to compute the Pickcands constants. This application will be discussed in Remark 3 .

The rest of this paper is organized as follows. In Section 2, we present the problem settings and some existing results that we will refer to in the later analysis. Section 3 presents the Monte Carlo methods and their efficiency results. Numerical implementations are included in Section 4 . Sections 5, 6, and 7 include the proofs of the theorems. 


\section{Preliminaries: Gaussian random fields and rare-event simula- tion}

\subsection{Gaussian random fields}

Throughout this paper, we consider a Gaussian random field living on a $d$-dimensional compact subset $T \subset R^{d}$, that is, for any finite subset $\left(t_{1}, \ldots, t_{n}\right) \subset T,\left(f\left(t_{1}\right), \ldots, f\left(t_{n}\right)\right)$ is a multivariate Gaussian random vector. For each $s, t \in T$, we define the following functions,

$$
\begin{aligned}
& \mu(t)=E(f(t)), \quad C(s, t)=\operatorname{Cov}(f(s), f(t)), \quad \mu_{T}=\sup _{t \in T}|\mu(t)|, \\
& \sigma^{2}(t)=C(t, t), \quad \sigma_{T}^{2}=\sup _{t \in T} \sigma^{2}(t), \quad r(s, t)=\frac{C(s, t)}{\sigma(s) \sigma(t)} .
\end{aligned}
$$

In this paper, we are mostly interested in the high excursion probability

$$
w(b)=P(M>b) .
$$

In addition to the tail probabilities, we also present the analysis concerning the integrals on the excursion set. Let $A_{\gamma}$ be the excursion set over the level $\gamma$

$$
A_{\gamma}=\{t \in T: f(t)>\gamma\}
$$

We define the integral

$$
\alpha(b)=\int_{A_{b}} \xi(t) d t
$$

where $\xi(t)$ is another random field living on $T$. Then we are interested in computing the conditional expectation

$$
v(b)=E(\alpha(b) \mid M>b)
$$

We now state the technical conditions that require the following definition.

Definition 2 A function $L$ is said to be slowly varying at zero if

$$
\lim _{x \rightarrow 0} \frac{L(t x)}{L(x)}=1, \quad \text { for all } t \in(0,1) .
$$

Throughout this paper, we impose the following technical conditions.

A1 The process $f(t)$ is almost surely continuous in $t$.

A2 For some $\alpha_{1} \in(0,2]$, the correlation function satisfies the following local expansion

$$
1-r(s, t) \sim \Delta_{s} L_{1}(|t-s|)|t-s|^{\alpha_{1}}, \quad \text { as } t \rightarrow s
$$

where $\Delta_{s} \in(0, \infty)$ is continuous in $s$ and $L_{1}$ is a slowly varying function at zero. Furthermore, there exist nonnegative constants $\kappa_{r}, \beta_{0}$, and positive constant $\beta_{1}>0$ satisfying $\beta_{0}+\beta_{1} \geq \alpha_{1}$ such that

$$
\left|r\left(t, t+s_{1}\right)-r\left(t, t+s_{2}\right)\right| \leq \kappa_{r} L_{1}\left(\left|s_{1}\right|\right)\left|s_{1}\right|^{\beta_{0}}\left|s_{1}-s_{2}\right|^{\beta_{1}} \quad \text { for }\left|s_{1}\right| \leq\left|s_{2}\right| .
$$

A3 The correlation function is non-degenerate, that is, $r(s, t)<1$ for all $s \neq t$. 
A4 The standard deviation $\sigma(t)$ belongs to either of the following two types.

Type $1 \sigma(t)=1$ for all $t \in T$.

Type $2 \sigma(t)$ has a unique maximum attained at $t^{*}$ satisfies the following conditions

$$
\begin{array}{rlrl}
|\sigma(t)-\sigma(s)| & \leq \kappa_{\sigma} \times L_{2}(|t-s|) \times|t-s|^{\alpha_{2}} & & \text { for all } s, t \in T \\
\sigma\left(t^{*}\right)-\sigma(t) & \sim \Lambda \times L_{2}\left(\left|t-t^{*}\right|\right) \times|t-t|^{\alpha_{2}} & \text { as } t \rightarrow t^{*}
\end{array}
$$

where $\alpha_{2} \in(0,1], \Lambda>0$, and $L_{2}$ is a slowly varying function at zero such that the limit $\lim _{x \rightarrow 0+} \frac{L_{1}(x)}{L_{2}(x)}$ exists.

A5 There exists $\kappa_{\mu}>0$ such that if $\sigma(t)$ is of Type 1 then $|\mu(s)-\mu(s+t)| \leq \kappa_{\mu} \sqrt{L_{1}(|t|)}|t|^{\alpha_{1} / 2}$; if $\sigma(t)$ is of Type 2 then $|\mu(s)-\mu(s+t)| \leq \kappa_{\mu} \sqrt{L_{2}(|t|)}|t|^{\alpha_{2} / 2}$.

A6 There exist $\kappa_{m}$ and $\epsilon$ small enough, such that mes $(B(t, \epsilon) \cap T) \geq \kappa_{m} \epsilon^{d} \omega_{d}$, for any $t \in T$, where $B(t, \epsilon)$ is the $\epsilon$-ball centered around $t$ and $\omega_{d}$ is the volume of the $d$-dimensional unit ball.

Condition A2 ensures that the normalized process $\frac{f(t)-\mu(t)}{\sigma(t)}$ is Hölder continuous with coefficient $\alpha_{1} / 2$. The bound in (8) imposes slightly more conditions. For instance, in case when $1-r(s, t)=$ $|t-s|^{\alpha_{1}}$, we can choose that $\beta_{0}=\alpha_{1}-1$ and $\beta_{1}=1$ if $\alpha_{1} \geq 1 ; \beta_{0}=0$ and $\beta_{1}=\alpha_{1}$ if $0<\alpha_{1}<1$. Condition A3 excludes the degenerated cases that are not essential and it makes the technical development more concise. Conditions A4 and A5 require that the mean and the standard deviation functions are also Hölder continuous. In Condition A4, we can adjust the constant $\Lambda$ such that the limit $\lim _{x \rightarrow 0+} L_{1}(x) / L_{2}(x)$ belongs to the set $\{0,1, \infty\}$. Condition A5 ensures that the variation of the mean function is bounded by those of $f(t)$ and $\sigma(t)$. In the later technical developments, the analysis is divided into two cases $\alpha_{1}<\alpha_{2}$ and $\alpha_{1} \geq \alpha_{2}$.

Throughout this paper, we use the following notations for the asymptotics. We write $h(b)=$ $o(g(b))$ if $h(b) / g(b) \rightarrow 0$ as $b \rightarrow \infty ; h(b)=O(g(b))$ if $h(b) \leq \kappa g(b)$ for some $\kappa>0 ; h(b)=\Theta(g(b))$ if $h(b)=O(g(b))$ and $g(b)=O(h(b)) ; h(b) \sim g(b)$ if $h(b) / g(b) \rightarrow 1$ as $b \rightarrow \infty$.

\subsection{Rare-event simulation and importance sampling}

\subsubsection{Rare-event simulation.}

The research focus of rare-event simulation is on estimating $w=P(B)$, where $P(B) \approx 0$. It is customary to introduce a parameter, say $b>0$, with a meaningful interpretation from an applied standpoint such that $w(b) \rightarrow 0$ as $b \rightarrow \infty$. Consider an estimator $Z_{b}$ such that $E Z_{b}=w(b)$. A popular efficiency concept in the rare-event simulation literature is the so-called strong efficiency that is defined as follows (c.f. [5, 13, 16]).

Definition 3 A Monte Carlo estimator $Z_{b}$ is said to be strongly efficient in estimating $w(b)$ if $E\left(Z_{b}\right)=w(b)$ and there exists a $\kappa_{0} \in(0, \infty)$ such that

$$
\sup _{b>0} \frac{\operatorname{Var}\left(Z_{b}\right)}{w^{2}(b)}<\kappa_{0}
$$

Strong efficiency measures mean squared error in relative terms for an unbiased estimator. Suppose that a strongly efficient estimator of $w(b)$ has been constructed, denoted by $Z_{b}$, and $n$ i.i.d. replicates 
of $Z_{b}$ are generated $Z_{b}^{(1)}, \ldots, Z_{b}^{(n)}$. Let

$$
\bar{Z}_{b, n} \triangleq \frac{1}{n} \sum_{i=1}^{n} Z_{b}^{(i)}
$$

be the averaged estimator that has an variance

$$
\operatorname{Var}\left(\bar{Z}_{b, n}\right)=\frac{\operatorname{Var}\left(Z_{b}\right)}{n} .
$$

By means of the Chebyshev's inequality, we obtain that

$$
P\left(\left|\bar{Z}_{b, n}-w(b)\right|>\varepsilon w(b)\right) \leq \frac{\operatorname{Var}\left(Z_{b}\right)}{n \varepsilon^{2} w^{2}(b)} .
$$

For any $\delta>0$, to achieve the $\varepsilon-\delta$ accuracy, we need to generate

$$
n=\frac{\operatorname{Var}\left(Z_{b}\right)}{\delta \varepsilon^{2} w^{2}(b)} \leq \frac{\kappa_{0}}{\delta \varepsilon^{2}}
$$

replicates of $Z_{b}$. This choice of $n$ is uniform in the rarity parameter $b$. We will later show that the proposed continuous importance sampling estimator is strongly efficient. Besides strong efficiency, another weaker concept is the so-called weak/asymptotic efficiency, that is,

$$
\lim _{b \rightarrow \infty} \frac{\log \operatorname{Var}\left(Z_{b}\right)}{2 \log w(b)}=1
$$

Weak efficiency implies that $\operatorname{Var}\left(Z_{b}\right)=o\left(w(b)^{\varepsilon}\right)$ for any $\varepsilon>0$.

\subsubsection{Importance sampling and variance reduction.}

Importance sampling is based on the basic identity,

$$
P(B)=\int I(\omega \in B) d P(\omega)=\int I(\omega \in B) \frac{d P}{d Q}(\omega) d Q(\omega) \quad \text { for a measurable set } B,
$$

where we assume that the probability measure $Q$ is such that $Q(\cdot \cap B)$ is absolutely continuous with respect to the measure $P(\cdot \cap B)$. If we use $E^{Q}$ to denote expectation under $Q$, then (11) trivially yields that the random variable

$$
Z(\omega)=I(\omega \in B) \frac{d P}{d Q}(\omega)
$$

is an unbiased estimator of $P(B)>0$ under the measure $Q$, or symbolically, $E^{Q} Z=P(B)$.

A central component lies in the selection of $Q$ in order to minimize the variance of $Z$. It is easy to verify that if we choose $\mathcal{Q}^{*}(\cdot)=P(\cdot \mid B)=P(\cdot \cap B) / P(B)$ then the corresponding estimator has zero variance and thus it is usually referred to as the the zero-variance change of measure. However, $\mathcal{Q}^{*}$ is clearly a change of measure that is of no practical value, since $P(B)$ - the quantity that we are attempting to evaluate in the first place - is unknown. Nevertheless, when constructing a good importance sampling distribution for a family of sets $\left\{B_{b}: b \geq b_{0}\right\}$ for which $0<P\left(B_{b}\right) \rightarrow 0$ as $b \rightarrow \infty$, it is often useful to analyze the asymptotic behavior of $\mathcal{Q}^{*}$ as $P\left(B_{b}\right) \rightarrow 0$ in order to guide the construction of a useful $Q$.

We now describe briefly how an efficient importance sampling estimator for $P\left(B_{b}\right)$ can also be 
used to estimate a large class of conditional expectations given $B_{b}$. Suppose that an importance sampling estimator has been constructed

$$
Z_{b} \triangleq I\left(\omega \in B_{b}\right) \frac{d P}{d Q}
$$

such that $\operatorname{Var}\left(L_{b}\right)=O\left(P\left(B_{b}\right)^{2}\right)$. Then, by noting that

$$
\frac{E^{Q}\left(X Z_{b}\right)}{E^{Q}\left(Z_{b}\right)}=\frac{E\left[X ; B_{b}\right]}{P\left(B_{b}\right)}=E\left[X \mid B_{b}\right]
$$

it follows easily that an estimator can be naturally obtained; i.e. the ratio of the corresponding averaged importance sampling estimators suggested by the ratio in the left of (12). Of course, when $X$ is difficult to simulate exactly, one must assume that the bias in estimating $E\left[X ; B_{b}\right]$ can be reduced with certain computational costs.

\subsubsection{The bias control.}

In addition to the variance control, one also needs to account for the computational effort required to generate $Z_{b}$. This issue is especially important for the current study. The random objects in this analysis are continuous processes. For the implementation, we need to use a discrete object to approximate the continuous process. Inevitably, the discretization induces bias, though it vanishes as the size of the discretization increases. To ensure the $\varepsilon-\delta$ relative accuracy, the bias needs to be controlled to a level less than $\varepsilon w(b)$.

The discretized estimators in [2] can be shown to be weakly efficient for general uniformly Hölder continuous Gaussian processes and it is strongly efficient when the process is twice differentiable and homogeneous. The analysis of the Hölder continuous fields relies heavily on the discrete nature of the estimators. For the implementation, it is established that, to ensure a bias of order $\varepsilon w(b)$, the size of the discretization must grow at a polynomial rate of $b$ for both differentiable and nondifferentiable fields. The authors also provide an optimality result. For twice differentiable and homogeneous fields, the size of a prefixed/deterministic set $T_{m}$ must be at least of order $O\left(b^{d}\right)$ so that the bias can be controlled to level $\varepsilon w(b)$.

\section{Main results}

The main results of this paper consist of the construction of a change of measure on the continuous sample path space on $T$, a random discretization scheme of $T$ associated with the change of measure $Q_{b}$, and lastly the efficiency results including the strong efficiency of the continuous and the discrete estimators and the complexity analysis of the discretization scheme.

\subsection{The change of measure}

As discussed previously, a key element of the analysis is the construction of a change of measure $Q_{b}$ (indexed by the rarity parameter $b$ ) that approximates the conditional measure $P(f \in \cdot \mid M>b)$. We should be able to compute the Radon-Nikodym derivative and also be able to simulate the process $f$ under $Q_{b}$. We describe the measure $Q_{b}$ from two aspects. First, we present its RadonNikokym derivative with respect to $P$

$$
\frac{d Q_{b}}{d P}(f)=\int_{T} h_{b}(t) \frac{q_{b, t}(f(t))}{\varphi_{t}(f(t))} d t
$$


where $h_{b}(t)$ is a density function on the set $T, q_{b, t}(x)$ is a density function on the real line, and $\varphi_{t}(x)$ is the density function of $f(t)$ under the measure $P$ evaluated at $f(t)=x$. We will need to choose $h_{b}(t)$ and $q_{b, t}(x)$ such that the measure $Q_{b}$ satisfies the absolute continuity condition to guarantee the unbiasedness.

We will present the specific forms of $h_{b}(t)$ and $q_{b, t}(x)$ momentarily. Before that, we would like to complete the description of $Q_{b}$ by presenting the simulation method of $f$ under $Q_{b}$.

Algorithm 1 (Continuous simulation) To generate a random sample path under the measure $Q_{b}$, we need a three-step procedure.

Step 1. Generate a random index $\tau \in T$ following the density $h_{b}(t)$.

Step 2. Conditional on the realization of $\tau$, sample $f(\tau)$ from the density $q_{b, \tau}(x)$.

Step 3. Conditional on the realization of $(\tau, f(\tau))$, generate $\{f(t): t \neq \tau\}$ from the original conditional distribution $P(f \in \cdot \mid f(\tau))$.

It is not difficult to verify that the above three-step procedure is consistent with the RandonNikodym derivative given as in (13). In particular, a random index $\tau$ is first sampled according to the density $h_{b}(t)$. Second, the random field at the location $\tau, f(\tau)$, is sampled from the distribution $q_{b, \tau}(x)$. Lastly, the rest of the field is sampled according the nominal/original condition distribution. The process $f(t)$ mostly follows the distribution under $P$ except at one random location $\tau$ where the process is twisted to follow an alternative distribution $q_{b, \tau}(x)$. Therefore, the overall RandonNikodym derivative is an average of the likelihood ratio $q_{b, t}(f(t)) / \varphi_{t}(f(t))$ with respect to the density $h_{b}(t)$.

Now, we present the specific forms of $h_{b}(t)$ and $q_{b, t}(x)$ for the computation of $w(b)$. For some positive constant $a$, let $\gamma$ be

$$
\gamma=b-a / b
$$

We choose

$$
q_{b, t}(x)=\varphi_{t}(x) \frac{I(f(t)>\gamma)}{P(f(t)>\gamma)}
$$

that is the conditional distribution of $f(t)$ given that $f(t)>\gamma$. The distribution of $\tau$ is chosen to be

$$
h_{b}(t)=\frac{P(f(t)>\gamma)}{\int_{T} P(f(t)>\gamma) d t} .
$$

The choice of $a$ in (14) does not affect the efficiency results, nor the complexity analysis. To simplify the discussion, we fix $a$ to be unity, that is,

$$
\gamma=b-1 / b
$$

As we explained previously, the random index $\tau$ indicates the location where the random field is twisted. Furthermore, $q_{b, t}(x)$ is chosen to be the conditional distribution given a high excursion. We emphasize that it is necessary to set $\gamma$ slightly lower than the target level $b$. This will technically provide a stochastic bound on the distribution of the likelihood ratio. The index $\tau$ basically localizes the maximum of $f(t)$. Note that $\tau$ is not necessarily, but is very close to, $t_{*} \triangleq \arg \sup f(t)$. Thus, as an approximation of the zero-variance change of measure, the distribution $h_{b}(t)$ should be chosen close to the conditional distribution of the maximum $t_{*}$ given that $f\left(t_{*}\right)>b$. This is our guideline 
to choose $h_{b}(t)$. For each $t \in T$, the conditional probability that $f(t)>b$ given $M>b$ is

$$
P(f(t)>b \mid M>b)=\frac{P(f(t)>b)}{P(M>b)} .
$$

The denominator $P(M>b)$ is free of $t$ and thus $P(f(t)>b \mid M>b) \propto P(f(t)>b)$. Our choice of $h_{b}(t) \propto P(f(t)>\gamma)$ approximates $P(f(t)>b \mid M>b)$ by replacing $b$ with $\gamma$ which is a very minor twist. This twist allows quite a lot of technical convenience. With such choices of $h_{b}(t)$ and $q_{b, t}(x)$, the Radon-Nikodym takes the following form

$$
\frac{d Q_{b}}{d P}=\frac{\int_{T} I(f(t)>\gamma) d t}{\int_{T} P(f(t)>\gamma) d t}=\frac{\operatorname{mes}\left(A_{\gamma}\right)}{\int_{T} P(f(t)>\gamma) d t},
$$

where mes $(\cdot)$ is the Lebesgue measure. According to Fubini's theorem, the denominator has another representation:

$$
\int_{T} P(f(t)>\gamma) d t=E\left[\operatorname{mes}\left(A_{\gamma}\right)\right]
$$

Remark 1 For different problems, we may choose different $h_{b}(t)$ and $q_{b, t}(x)$ to approximate various conditional distributions. For instance, $q_{b, t}(x)$ was chosen to be in the exponential family of $\varphi_{t}(x)$ in [20] for the derivation of tail approximations of $\int e^{f(t)} d t$.

\subsection{An adaptive discretization scheme and the algorithms}

\subsubsection{The continuous estimator and the challenges}

Based on the change of measure $Q_{b}$, a natural estimator for $w(b)$ is given by

$$
Z_{b} \triangleq I(M>b) \frac{d P}{d Q_{b}}=I(M>b) \frac{\int_{T} P(f(t)>\gamma) d t}{m e s\left(A_{\gamma}\right)} .
$$

It is straightforward to obtain that $E_{b}\left(Z_{b}\right)=w(b)$, where we use $E_{b}(\cdot)$ to denote the expectation under the measure $Q_{b}$. The second moment of $Z_{b}$ is given by

$$
E_{b}\left(Z_{b}^{2}\right)=E_{b}\left[\frac{\left(\int_{T} P(f(t)>\gamma) d t\right)^{2}}{m e s^{2}\left(A_{\gamma}\right)} ; M>b\right] .
$$

We will later show that this continuous estimator (under regularity conditions) is strongly efficient, that is, $E_{b}\left(Z_{b}^{2}\right)=O\left(w^{2}(b)\right)$. Similarly, a natural estimator for the numerator $E(\alpha(b) ; M>b)$ in (12) is

$$
Y_{b} \triangleq \frac{\alpha(b)}{m e s\left(A_{\gamma}\right)} \int_{T} P(f(t)>\gamma) d t
$$

which, under regularity conditions, will be shown to estimate $E(\alpha(b) ; M>b)$ with strong efficiency.

For the implementation, we are not able to simulate the continuous field $f$ and therefore have to adopt a simulatable estimator, $\hat{Z}_{b}$, that approximates the continuous estimator $Z_{b}$. A natural approach is to consider the random field on a finite set $T_{m}=\left\{t_{1}, \ldots, t_{m}\right\} \subset T$ and use $P\left(\max _{T_{m}} f\left(t_{i}\right)>b\right)$ as an approximation of $w(b)=P\left(\sup _{T} f(t)>b\right)$. The bias is given by

$$
P\left(\sup _{T} f(t)>b\right)-P\left(\max _{T_{m}} f(t)>b\right)=P\left(T_{m} \cap A_{b}=\emptyset, M>b\right) .
$$


In what follows, we explain without rigorous derivation that the above scheme usually induces a heavy computational overhead. To simplify the discussion, we consider the special case that $f$ is a stationary process and its covariance function satisfies the local expansion (slightly abusing the notation)

$$
C(t) \triangleq \operatorname{Cov}(f(s), f(s+t))=1-|t|^{\alpha}+o\left(|t|^{\alpha}\right)
$$

Then, the process is Hölder continuous with coefficient $\alpha / 2$. Under this setting, standard results yield the following estimate of the excursion set

$$
E\left(\operatorname{mes}\left(A_{b}\right) \mid M>b\right)=\Theta\left(b^{-2 d / \alpha}\right)
$$

Thanks to stationarity, conditional on the event $\{M>b\}$, the excursion set $A_{b}$ is a random subset of $T$, whose volume is of order $\Theta\left(b^{-2 d / \alpha}\right)$ and which is approximately uniformly distributed over the domain $T$.

Notice that the bias term $P\left(T_{m} \cap A_{b}=\emptyset, M>b\right)$ is the probability that $T_{m}$ does not intersect with $A_{b}$. Therefore, if $m \ll b^{2 d / \alpha}, T_{m}$ is too sparse such that it is not able to catch the set $A_{b}$ no matter how $T_{m}$ is distributed over $T$. Therefore, it is necessary to have a lattice of size at least of order $O\left(b^{2 d / \alpha}\right)$. This heuristic calculation was made rigorous for smooth fields in [2]. Thus, the computational complexity to generate the process $f$ on the set $T_{m}$ grows at a polynomial rate with $b$. In this paper, we aim at further reduction of the discretization size to a constant level while still maintaining the $\varepsilon$-relative bias. For this sake, we need to seek among the random discrete sets.

\subsubsection{A closer look at the excursion set $A_{\gamma}$}

The proposed adaptive discretization scheme is closely associated with the three step simulation procedure under $Q_{b}$ and furthermore the distribution of $A_{\gamma}$. Among the three steps in Algorithm 1. Step 1 and Step 2 are implementable. It is Step 3, generating $\{f(t): t \neq \tau\}$ conditional on $(\tau, f(\tau))$, that requires discretization. In order to estimate $w(b)$ and to generate the estimator $Z_{b}$, we only need to simulate the random indicator $I(M>b)$ and the volume of the excursion set mes $\left(A_{\gamma}\right)$ conditional on $(\tau, f(\tau))$. The term $\int_{T} P(f(t)>\gamma) d t$ is a deterministic number that can be computed via routine numerical methods.

In what follows, we focus on the simulation and approximation of $I(M>b)$ and $m e s\left(A_{\gamma}\right)$. For illustration purpose, we provide the discussion for the homogeneous case with covariance function satisfying the expansion (21). We define $\zeta=b^{2 / \alpha}$ that characterizes the cluster size of $f$. Furthermore, we define the normalized process

$$
g(t)=b(f(\tau+t / \zeta)-b)
$$

Note that $b \times(f(\tau)-\gamma)$ asymptotically follows an exponential distribution. Conditional on $f(\tau)=$ $\gamma+z / b$ the $g$ process has expectation

$$
E_{b}[g(t) \mid f(\tau)=\gamma+z / b]=z-1-(1+o(1))|t / \zeta|^{\alpha}\left[b^{2}+(z-1)\right] .
$$

For all $z=o\left(b^{2}\right)$, we have that

$$
E_{b}[g(t) \mid f(\tau)=\gamma+z / b]=z-1-(1+o(1))|t|^{\alpha} \quad \text { as } b \rightarrow \infty .
$$

In addition, the covariance of $g(t)$ is

$$
\operatorname{Cov}(g(s), g(t))=\left(|s|^{\alpha}+|t|^{\alpha}-|s-t|^{\alpha}\right)+o(1)
$$


where $o(1) \rightarrow 0$ as $b \rightarrow \infty$. Therefore, the distribution of $g(t)$ converges weakly to a Gaussian process with the above mean and covariance function. In addition, $f(\tau+t / \zeta) \geq \gamma$ if and only if $g(t)>0$. The excursion set $A_{\gamma}$ can be written as

$$
A_{\gamma}=\tau+\zeta^{-1} \cdot A_{-1}^{g} \triangleq\left\{\tau+\zeta^{-1} t: t \in A_{0}^{g}\right\} .
$$

where $A_{-1}^{g}=\{t: g(t)>-1\}$. Note that the process $g(t)$ is a Gaussian process with standard deviation $O\left(|t|^{\alpha / 2}\right)$ and a negative drift of order $O\left(-|t|^{\alpha}\right)$. Therefore, in expectation, $g(t)$ goes below 0 when $z \ll|t|^{\alpha}$ where $z$ is asymptotically an exponential random variable. Thus, the excursion set $A_{-1}^{g}$ is of order $O(1)$. Furthermore, $A_{\gamma}$ is a random set within $O\left(\zeta^{-1}\right)$ distance from the random index $\tau$. The volume mes $\left(A_{\gamma}\right)$ is of order $O\left(\zeta^{-d}\right)$. The above discussion quantifies the intuition that $\tau$ localizes the global maximum of $f$. It also localizes the excursion set $A_{\gamma}$. Therefore, upon considering approximating/computing mes $\left(A_{\gamma}\right)$ and $I(M>b)$, we should focus on the region around $\tau$.

Conditional on a specific realization of the process $f$, we formulate the approximation of $\operatorname{mes}\left(A_{\gamma}\right)$ as estimation problem. Note that the ratio $\operatorname{mes}\left(A_{\gamma}\right) / \operatorname{mes}(T) \in[0,1]$ corresponds to the following probability

$$
\frac{\operatorname{mes}\left(A_{\gamma}\right)}{\operatorname{mes}(T)}=P\left(U \in A_{\gamma}\right)
$$

where $U$ is a uniform random variable on the set $T$ with respect to the Lebesgue measure. Estimating mes $\left(A_{\gamma}\right)$ constitutes another rare-event simulation problem.

\subsubsection{An adaptive discretization scheme.}

Based on the understanding of the excursion set $A_{\gamma}$, we setup a discretization scheme adaptive to the realization of $\tau$. To proceed, we provide the general form of $\zeta$ in presence of slowly varying functions

$$
\zeta \triangleq \max \left\{|s|^{-1}: L_{1}(|s|)|s|^{\alpha_{1}} \geq b^{-2} \text { or } L_{2}(|s|)|s|^{\alpha_{2}} \geq b^{-2}\right\} .
$$

In the case of constant variance, we formally define $\alpha_{2}=\infty$ and thus $\zeta$ is defined as $\zeta \triangleq \max \left\{|s|^{-1}\right.$ : $\left.L_{1}(|s|)|s|^{\alpha_{1}} \geq b^{-2}\right\}$. To facilitate the later discussion, we define two other scale factors

$$
\zeta_{i} \triangleq \max \left\{|s|^{-1}: L_{i}(|s|)|s|^{\alpha_{i}} \geq b^{-2}\right\}, \quad i=1,2 .
$$

Thus, it is straightforward to verify that

$$
\zeta=\max \left(\zeta_{1}, \zeta_{2}\right)
$$

Consider an isotropic distribution (centered around zero) with density $k(t)$, that is, $k(t)=k(s)$ if $|s|=|t|$. We choose $k(t)$ to be reasonably heavy-tailed such that for some $\varepsilon_{1}>0$

$$
k(t) \sim|t|^{-d-\varepsilon_{1}}, \quad \text { as } t \rightarrow \infty .
$$

In addition there exists a $\kappa_{1}>0$ such that $k(t) \leq \kappa_{1}$ for all $t$. For instance, we can choose $k(t)$ to be, but not necessarily restricted to, the multivariate $t$-distribution. Furthermore, conditional on $\tau$, we define the rescaled density

$$
k_{\tau, \zeta}(t)=\zeta^{d} \times k(\zeta(t-\tau))
$$


that centers around $\tau$ and has scale $\zeta^{-1}$. We construct a $\tau$-adapted random subset of $T$ by generating i.i.d. random variables from the density $k_{\tau, \zeta}(t)$, denoted by $t_{1}, \ldots, t_{m}$. Then, define

$$
\widehat{m e s}\left(A_{\gamma}\right) \triangleq \frac{1}{m} \sum_{i=1}^{m} \frac{I\left(f\left(t_{i}\right)>\gamma\right)}{k_{\tau, \zeta}\left(t_{i}\right)}
$$

that is an unbiased estimator of $m e s\left(A_{\gamma}\right)$ in the sense that for each realization of $f$

$$
E_{\tau, \zeta}\left[\widehat{m e s}\left(A_{\gamma}\right) \mid f\right]=\operatorname{mes}\left(A_{\gamma}\right)
$$

where $E_{\tau, \zeta}(\cdot \mid f)$ is the expectation with respect to $t_{1}, \ldots, t_{m}$ under the density $k_{\tau, \zeta}$ for a particular realization of $f$. Notationally, if $t_{i} \notin T$, then $I\left(f\left(t_{i}\right)>\gamma\right)=0$.

Similar to the approximation of mes $\left(A_{\gamma}\right)$, we use the same $\tau$-adapted random subset to approximate $I(M>b)$, that is,

$$
I\left(\max _{i=1}^{\max } f\left(t_{i}\right)>b\right) \approx I(M>b) .
$$

Based on the above discussions, we present the final algorithm.

Algorithm 2 The algorithm consists of the following steps.

Step 1. Generate a random index $\tau \in T$ following the density $h_{b}(t)$ in (16).

Step 2. Conditional on the realization of $\tau$, sample $f(\tau)$ from $q_{b, t}(x)$ in (15).

Step 3. Conditional on the realization of $\tau$, generate i.i.d. random indices $t_{1}, \ldots, t_{m}$ following density $k_{\tau, \zeta}(t)$.

Step 4. Conditional on the realization of $(\tau, f(\tau))$, generate multivariate normal random vector $\left(f\left(t_{1}\right), \ldots, f\left(t_{m}\right)\right)$ from the original/nominal conditional distribution of $P(\cdot \mid f(\tau))$.

Step 5. Output

$$
\hat{Z}_{b}=\frac{I\left(\max _{i=1}^{m} f\left(t_{i}\right)>b\right)}{\widehat{m e s}\left(A_{\gamma}\right)} \int_{T} P(f(t)>\gamma) d t
$$

where $\widehat{m e s}\left(A_{\gamma}\right)$ is given as in (26).

For the discrete version of the estimator $Y_{b}$ as in (20), we approximate it in a similar way. In Step 4 of the above algorithm, we simulate $\left\{\left(f\left(t_{i}\right), \xi\left(t_{i}\right)\right): i=1, \ldots, m\right\}$ jointly conditional on $(\tau, f(\tau))$. Then, we output the estimator

$$
\hat{Y}_{b}=\frac{\hat{\alpha}\left(A_{b}\right)}{\widehat{m e s}\left(A_{\gamma}\right)} \int_{T} P(f(t)>\gamma) d t
$$

where

$$
\hat{\alpha}\left(A_{b}\right) \triangleq \frac{1}{m} \sum_{i=1}^{m} \frac{\xi\left(t_{i}\right)}{k_{\tau, \zeta}\left(t_{i}\right)} I\left(f\left(t_{i}\right)>b\right) .
$$

\subsection{The main results}

We present the efficiency results of the proposed algorithms. The first theorem establishes that the continuous estimator is strongly efficient. 
Theorem 1 Consider a Gaussian random field $f$ that satisfies conditions A1-6. Let $Z_{b}$ be given as in (19) and Algorithm 1. Then, $Z_{b}$ is strongly efficient in estimating $w(b)$, that is, there exists $\kappa_{0}$ such that

$$
E_{b}\left(Z_{b}^{2}\right) \leq \kappa_{0} w^{2}(b)
$$

for all $b>0$.

The next theorem establishes the computation complexity of the discrete estimator.

Theorem 2 Consider a Gaussian random field $f$ that satisfies conditions A1-6. Let $\hat{Z}_{b}$ be the estimator given by Algorithm Q Q. There exists $\lambda>0$ such that for any $\varepsilon>0$ if we choose $m=$ $\lambda \varepsilon^{-d\left(2 / \min \left(\alpha_{1}, \alpha_{2}\right)+2 / \beta_{1}\right)}$, then

$$
\left|E_{b}\left(\hat{Z}_{b}\right)-w(b)\right| \leq \varepsilon w(b)
$$

for all $b>0$. Furthermore, there exists $\kappa_{0}$ such that

$$
E_{b}\left(\hat{Z}_{b}^{2}\right) \leq \kappa_{0} w^{2}(b) .
$$

With the above results, we generate $n$ i.i.d. replicates of $\hat{Z}_{b}$, denoted by $\hat{Z}_{b}^{(1)}, \ldots, \hat{Z}_{b}^{(n)}$, with $m$ chosen as in the theorem such that the averaged estimator, $\frac{1}{m} \sum_{i=1}^{n} \hat{Z}_{b}^{(i)}$, has its bias bounded by $\varepsilon w(b) / 2$ and its variance is bounded by $\kappa_{0} w^{2}(b) / n$. To achieve $\varepsilon$ relative error with at $(1-\delta)$ confidence, we need to choose $n=\frac{4 \kappa_{0}}{\varepsilon^{2} \delta}$, that is,

$$
P\left(\left|\frac{1}{m} \sum_{i=1}^{n} \hat{Z}_{b}^{(i)}-w(b)\right|>\varepsilon w(b)\right)<\delta
$$

and the total computational complexity is of order $O\left(m^{3} \varepsilon^{-2} \delta^{-1}\right)$, where $m^{3}$ is the complexity of computing the eigenvalue of an $m \times m$ covariance matrix.

Theorem 3 Consider a Gaussian random field $f$ that satisfies conditions A1-6. There exists $0<a_{1}<a_{2}<\infty$, such that $\xi(t) \in\left[a_{1}, a_{2}\right]$ almost surely. We have the following results

1. Then, there exists $\kappa_{0}$ such that for all $b>0$

$$
E_{b}\left(Y_{b}^{2}\right) \leq \kappa_{0} u^{2}(b)
$$

where $u(b)=E(\alpha(b) ; M>b)$.

2. There exists $\lambda$ such that for each $\varepsilon>0$ if we choose $m=\lambda \varepsilon^{-d\left(2 / \min \left(\alpha_{1}, \alpha_{2}\right)+2 / \beta_{1}\right)}$ then

$$
\left|E_{b}\left(\hat{Y}_{b}\right)-u(b)\right| \leq \varepsilon u(b)
$$

and

$$
E_{b}\left(\hat{Y}_{b}^{2}\right) \leq \kappa_{0} u^{2}(b)
$$

In the previous theorem, we require that the process $\xi(t)$ take values in a positive interval $\left[a_{1}, a_{2}\right]$. This constraint is imposed for technical convenience. There are several ways in which we can relax this condition. If $\xi(t)$ is independent of $f(t)$, then, we can relax the interval to be $(0, \infty)$. In the case when $\xi(t) \in(0, \infty)$ and $\xi(t)$ and $f(t)$ are dependent, we may need to modify the algorithm. This is because $\xi(t)$ could be very close to zero on the excursion set $A_{b}$ and therefore the estimator (27) may not be strongly efficient in estimating $\alpha(t)$. In this case, we may further 
change the sampling distribution of $\left\{\left(f\left(t_{i}\right), \xi\left(t_{i}\right)\right): i=1, \ldots, m\right\}$ to reduce the variance of $\hat{\alpha}(t)$. These modifications have to be case-by-case and they can be handled by routine variance reduction techniques that we do not pursue in this paper.

Remark 2 There are cases that the current setting does not cover. For instance, the process is anisotropic in the sense that $\alpha$ depends on the direction; see, for instance, 24 for more discussions. We believe that the results of Theorem 1 hold under this setting. We need to follow the same idea and apply our proof technique in different directions. For the discretization scheme, one needs to define the scale $\zeta$ for different directions and rescale the density $k(t)$ differently among different directions. Thus, we expect the results of Theorem 2 and Theorem 0 to hold.

Remark 3 The current work provides a means to numerically compute the Pickands constant. The basic idea is to numerically compute tail probability $w(b)$ for $b$ very large and for some stationary process living on $[0,1]$ with covariance function $C(t)=e^{-|t|^{\alpha}}$. Denote the estimate by $\hat{w}(b)$. Then, an estimate of the Pickands' constant is given by

$$
\hat{H}_{\alpha}=\frac{\hat{w}(b)}{b^{2 / \alpha} P(Z>b)} .
$$

\section{Numerical analysis}

In this section, we present four numerical examples to show the performance of our algorithms. First, we applied our algorithm to a one dimensional Gaussian field whose tail probability is in a closed form. For the discretization, we deploy $m=20$ points when $d=1$ and 40 points when $d=2$. To make sure that the bias is small enough, we have run the simulations with 10 times more points and the results didn't change substantially. We only report the results with fewer points to illustrate the efficiency.

Example 1 Consider $f(t)=X \cos t+Y \sin t, T=[0,3 / 4]$, where $X$ and $Y$ are independent standard Gaussian variables. The probability $P\left(\sup _{t \in T} f(f)>b\right)$ is known to be in closed form ([1]), and is given by

$$
P\left(\sup _{0 \leq t \leq 3 / 4} f(t)>b\right)=1-\Phi(b)+\frac{3}{8 \pi} e^{-b^{2} / 2} .
$$

Table 1 shows the simulation results.

\begin{tabular}{cccc}
\hline $\mathrm{b}$ & true value & est & std err \\
\hline 3 & $2.7 \mathrm{E}-03$ & $2.6 \mathrm{E}-03$ & $1.1 \mathrm{E}-04$ \\
4 & $7.2 \mathrm{E}-05$ & $7.2 \mathrm{E}-05$ & $3.2 \mathrm{E}-06$ \\
5 & $7.3 \mathrm{E}-07$ & $7.3 \mathrm{E}-07$ & $3.4 \mathrm{E}-08$ \\
6 & $2.8 \mathrm{E}-09$ & $2.8 \mathrm{E}-09$ & $1.4 \mathrm{E}-10$ \\
7 & $4.0 \mathrm{E}-12$ & $4.1 \mathrm{E}-12$ & $2.0 \mathrm{E}-13$ \\
8 & $2.2 \mathrm{E}-15$ & $2.1 \mathrm{E}-15$ & $8.4 \mathrm{E}-17$ \\
\hline
\end{tabular}

Table 1: Simulation results for the cosine process where $\mathrm{n}=1000, \mathrm{~m}=20, k(t)$ is chosen to be the density function of $t$-distribution with degrees of freedom 3. The "true value" is calculated from (28)

The following three examples treat random fields over a two dimensional square. 
Example 2 Consider a mean zero, unit variance, stationary and smooth Gaussian field over $T=$ $[0,1]^{2}$, with covariance function

$$
C(t)=e^{-|t|^{2}} .
$$

Let $\xi(t)=1$, then $E\left(\int_{A_{b}} \xi(t) d t\right)$ is in a closed form and is given by

$$
E\left(\int_{A_{b}} \xi(t) d t\right)=E\left(\operatorname{mes}\left(A_{b}\right)\right)=1-\Phi(b) .
$$

Table 4 shows the simulation results.

\begin{tabular}{ccccccc}
\hline & \multicolumn{2}{c}{$P\left(\sup _{T} f(t)>b\right)$} & & \multicolumn{3}{c}{$E\left(\operatorname{mes}\left(A_{b}\right)\right)$} \\
\cline { 2 - 3 } \cline { 5 - 7 } $\mathrm{b}$ & est & std err & & true value & est & std err \\
\hline 3 & $9.3 \mathrm{E}-03$ & $3.6 \mathrm{E}-04$ & & $1.3 \mathrm{E}-03$ & $1.4 \mathrm{E}-03$ & $4.0 \mathrm{E}-05$ \\
4 & $3.4 \mathrm{E}-04$ & $1.5 \mathrm{E}-05$ & & $3.2 \mathrm{E}-05$ & $3.3 \mathrm{E}-05$ & $9.2 \mathrm{E}-07$ \\
5 & $4.2 \mathrm{E}-06$ & $1.7 \mathrm{E}-07$ & & $2.9 \mathrm{E}-07$ & $3.0 \mathrm{E}-07$ & $8.2 \mathrm{E}-09$ \\
6 & $1.9 \mathrm{E}-08$ & $8.1 \mathrm{E}-10$ & & $9.9 \mathrm{E}-10$ & $1.0 \mathrm{E}-09$ & $2.8 \mathrm{E}-11$ \\
7 & $3.3 \mathrm{E}-11$ & $1.3 \mathrm{E}-12$ & & $1.3 \mathrm{E}-12$ & $1.4 \mathrm{E}-12$ & $3.7 \mathrm{E}-14$ \\
8 & $1.9 \mathrm{E}-14$ & $7.1 \mathrm{E}-16$ & & $6.7 \mathrm{E}-16$ & $6.7 \mathrm{E}-16$ & $1.8 \mathrm{E}-17$ \\
\hline
\end{tabular}

Table 2: Simulation results for Example 2, where $\mathrm{n}=1000, \mathrm{~m}=40 . \quad k(t)=\frac{25}{32 \pi}\left(1+0.64|t|^{2}\right)^{-3}$, the density function of multivariate $t$-distribution with degrees of freedom 4 , and $\mu=0 ; \Sigma=0.64 I_{2}$.

Example 3 Consider a continuous inhomogenous Gaussian field on $T=[0,1]^{2}$ with mean and covariance function

$$
\mu(t)=0.1 t_{1}+0.1 t_{2} \quad C(s, t)=e^{-|t-s|^{2}} .
$$

Let $\xi(t)=1$, then $E\left(\int_{A_{b}} \xi(t) d t\right)$ is in a closed form and is given by

$$
E\left(\int_{A_{b}} \xi(t) d t\right)=E\left(\operatorname{mes}\left(A_{b}\right)\right)=\int_{T} P(f(t)>b) d t .
$$

Table 3 shows the simulation results.

\begin{tabular}{ccccccc}
\hline & \multicolumn{2}{c}{$P\left(\sup _{T} f(t)>b\right)$} & & \multicolumn{3}{c}{$E\left(\right.$ mes $\left.\left(A_{b}\right)\right)$} \\
\cline { 2 - 3 } \cline { 5 - 7 } $\mathrm{n}$ & est & std err & & true value & est & std err \\
\hline 3 & $1.2 \mathrm{E}-02$ & $5.6 \mathrm{E}-04$ & & $1.9 \mathrm{E}-03$ & $1.8 \mathrm{E}-03$ & $5.4 \mathrm{E}-05$ \\
4 & $5.0 \mathrm{E}-04$ & $1.9 \mathrm{E}-05$ & & $4.8 \mathrm{E}-05$ & $5.0 \mathrm{E}-05$ & $1.4 \mathrm{E}-06$ \\
5 & $7.2 \mathrm{E}-06$ & $2.8 \mathrm{E}-07$ & & $4.9 \mathrm{E}-07$ & $5.1 \mathrm{E}-07$ & $1.4 \mathrm{E}-08$ \\
6 & $3.5 \mathrm{E}-08$ & $1.4 \mathrm{E}-09$ & & $1.9 \mathrm{E}-09$ & $1.9 \mathrm{E}-09$ & $5.4 \mathrm{E}-11$ \\
7 & $6.7 \mathrm{E}-11$ & $2.7 \mathrm{E}-12$ & & $2.7 \mathrm{E}-12$ & $2.6 \mathrm{E}-12$ & $7.7 \mathrm{E}-14$ \\
8 & $4.5 \mathrm{E}-14$ & $1.9 \mathrm{E}-15$ & & $1.5 \mathrm{E}-15$ & $1.5 \mathrm{E}-15$ & $4.3 \mathrm{E}-17$ \\
\hline
\end{tabular}

Table 3: Simulation result for Example3, where $\mathrm{n}=1000, \mathrm{~m}=40, k(t)$ is the same as that of Example 2 .

Example 4 Consider the continuous Gaussian field living on $T=[0,1]^{2}$ with mean and covariance function

$$
\mu(t)=0.1 t_{1}+0.1 t_{2} \quad C(s, t)=e^{-|t-s| / 4} .
$$


Let $\xi=1$ and then the true expectation is

$$
E\left(\int_{A_{b}} \xi(t) d t\right)=E\left(\operatorname{mes}\left(A_{b}\right)\right)=\int_{T} P(f(t)>b) d t .
$$

Table 4 shows the simulation results.

\begin{tabular}{ccccccc}
\hline & \multicolumn{2}{c}{$P\left(\sup _{T} f(t)>b\right)$} & & \multicolumn{3}{c}{$E\left(\right.$ mes $\left.\left(A_{b}\right)\right)$} \\
\cline { 2 - 3 } \cline { 5 - 7 } $\mathrm{b}$ & est & std err & & true value & est & std err \\
\hline 3 & $1.4 \mathrm{E}-02$ & $6.6 \mathrm{E}-04$ & & $1.9 \mathrm{E}-03$ & $1.9 \mathrm{E}-03$ & $5.3 \mathrm{E}-05$ \\
4 & $7.4 \mathrm{E}-04$ & $4.4 \mathrm{E}-05$ & & $4.9 \mathrm{E}-05$ & $5.1 \mathrm{E}-05$ & $1.4 \mathrm{E}-06$ \\
5 & $1.5 \mathrm{E}-05$ & $7.5 \mathrm{E}-07$ & & $4.9 \mathrm{E}-07$ & $5.1 \mathrm{E}-07$ & $1.4 \mathrm{E}-08$ \\
6 & $9.9 \mathrm{E}-08$ & $5.2 \mathrm{E}-09$ & & $1.9 \mathrm{E}-09$ & $1.9 \mathrm{E}-09$ & $5.4 \mathrm{E}-11$ \\
7 & $2.9 \mathrm{E}-10$ & $1.3 \mathrm{E}-11$ & & $2.7 \mathrm{E}-12$ & $2.7 \mathrm{E}-12$ & $7.8 \mathrm{E}-14$ \\
8 & $2.6 \mathrm{E}-13$ & $1.4 \mathrm{E}-14$ & & $1.5 \mathrm{E}-15$ & $1.5 \mathrm{E}-15$ & $4.3 \mathrm{E}-17$ \\
\hline
\end{tabular}

Table 4: Simulation result for Example 4, where $\mathrm{n}=1000, \mathrm{~m}=40, k(t)=\frac{1}{8 \pi}\left(1+|t|^{2}\right)^{-3}$, the density function of multivariate $t$-distribution, with degrees of freedom $4, \mu=0, \Sigma=4 I_{2}$.

For all the examples, the ratios of standard error over the estimated value do not increase as $b$ increase. This is consistent with our theoretical analysis. Also note that $m$ does not increase as the level increase, which reduces the computational complexity significantly. Overall, the numerical estimates are very accurate.

\section{Proof of Theorem 1}

Throughout the proof, we will use $\kappa$ as a generic notation to denote large and not-so-important constants whose value may vary from place to place. Similarly, we use $\varepsilon_{0}$ as a generic notation for small positive constants.

The first result we cite is the Borel-TIS (Borel-Tsirelson-Ibragimov-Sudakov) inequality [4, 11, 31 that will be used very often in our technical development.

Proposition 1 Let $f(t)$ be a centered Gaussian process almost surely bounded in $T$. Then,

$$
E\left[\sup _{t \in T} f(t)\right]<\infty
$$

and

$$
P\left(\sup _{t \in T} f(t)-E\left[\sup _{t \in T} f(t)\right] \geq b\right) \leq \exp \left(-b^{2} /\left(2 \sigma_{T}^{2}\right)\right) .
$$

In this proof we need to establish a lower bound of the probability

$$
w(b)=E_{b}\left[\frac{1}{m e s\left(A_{\gamma}\right)} ; M>b\right] \int_{T} P(f(t)>\gamma) d t
$$

and an upper bound of the second moment

$$
E_{b}\left(Z_{b}^{2}\right)=E_{b}\left[\frac{1}{m e s^{2}\left(A_{\gamma}\right)} ; M>b\right]\left[\int_{T} P(f(t)>\gamma) d t\right]^{2}
$$


The central analysis lies in the following two quantities:

$$
I_{1}=E_{b}\left[\frac{1}{\operatorname{mes}^{2}\left(A_{\gamma}\right)} ; M>b\right], \quad I_{2}=E_{b}\left[\frac{1}{m e s\left(A_{\gamma}\right)} ; M>b\right] .
$$

We will show that there exist constants $\kappa$ and $\varepsilon_{0}$ such that

$$
I_{1} \leq \kappa \zeta^{2 d}, \quad I_{2} \geq \varepsilon_{0} \zeta^{d} .
$$

If these inequalities are proved, then

$$
\limsup _{b \rightarrow \infty} \frac{I_{1}}{I_{2}^{2}}<\infty
$$

is in place, and we finish our proof for Theorem 1, For the rest of the proof, we establish these two inequalities.

To proceed, we describe the conditional Gaussian random field given $f(\tau)$. First, if we write $f(\tau)=\gamma+z / b$, then $z$ asymptotically follows an exponential distribution with expectation $\sigma^{2}(\tau)$. Conditional on $f(\tau)=\gamma+z / b$, let

$$
f(t+\tau)=E[f(t+\tau) \mid f(\tau)=\gamma+z / b]+f_{0}(t) .
$$

Thus, given $f(\tau), f_{0}(t)$ is a zero-mean Gaussian process. By means of conditional Gaussian calculation, the conditional mean and conditional covariance function are given by

$$
\begin{gathered}
\mu_{\tau}(t)=E(f(t+\tau) \mid f(\tau)=\gamma+z / b) \\
=\mu(t+\tau)+\frac{\sigma(\tau+t)}{\sigma(\tau)} r(\tau+t, \tau)(\gamma+z / b-\mu(\tau)) \\
C_{0}(s, t)=\operatorname{Cov}\left(f_{0}(s), f_{0}(t)\right) \\
=\sigma(\tau+s) \sigma(\tau+t)[r(s+\tau, t+\tau)-r(\tau+t, \tau) r(\tau+s, \tau)] .
\end{gathered}
$$

The next lemma controls the conditional variance.

Lemma 1 Under condition A1-6, there exists constants $\lambda_{1}>0$, such that for all $\tau \in T$, and $b$ large enough,

(i) for all $t+\tau \in T$,

$$
C_{0}(t, t) \leq \lambda_{1} L_{1}(|t|)|t|^{\alpha_{1}}
$$

(ii) for $s, t \in T$

$$
\operatorname{Var}\left(f_{0}(s)-f_{0}(t)\right) \leq \lambda_{1} \max \left(L_{1}(|t-s|)|t-s|^{\alpha_{1}}, L_{2}(|t-s|)|t-s|^{\alpha_{2}}\right) ;
$$

(iii) for any $\varepsilon>0$, there exists $\delta>0$ (independent of b) such that for each $t$

$$
E\left(\sup _{|s-t| \leq \delta \zeta^{-1}} f_{0}(s)\right)=\frac{\varepsilon}{b}
$$

The proofs for (i) and (ii) are an application of conditions A2, A3, A6, and elementary calculations. (iii) is a direct corollary of (ii) and Dudley's entropy bound (Theorem 1.1 of [15]). We omit the detailed derivations. We proceed to the analysis of $I_{1}$ and $I_{2}$ by considering the Type 1 and Type 2 standard deviations function (condition A4) separately. 
In the main text, we only provide the proof when $\sigma(t)$ is of Type 1 in Assumption A4, that is, a constant variance. The proof of the non-constant case is similar. We present it in the Supplemental Material. For the constant variance case that corresponds to $\alpha_{2}=\infty$, the scaling factor is given by

$$
\zeta=\zeta_{1}
$$

We aim at showing that $I_{1} \leq \kappa \zeta_{1}^{2 d}$ and $I_{2} \geq \varepsilon_{0} \zeta_{1}^{d}$.

\subsection{The $I_{1}$ term}

For some $y_{0}>0$ chosen to be sufficiently small (independent of $b$ ) and to be determined in the later analysis, the $I_{1}$ term is bounded by

$$
E_{b}\left[\frac{1}{m e s^{2}\left(A_{\gamma}\right)} ; M>b\right] \leq y_{0}^{-2 d} \zeta_{1}^{2 d}+E_{b}\left(\frac{1}{m e s^{2}\left(A_{\gamma}\right)} ; \operatorname{mes}\left(A_{\gamma}\right)<y_{0}^{d} \zeta_{1}^{d}, M>b\right),
$$

To control the second term of the above inequality, we need to provide a bound on the following tail probability for $0<y<y_{0}$

$$
\begin{aligned}
& Q\left(\operatorname{mes}\left(A_{\gamma}\right)<y^{d} \zeta_{1}^{-d}, M>b\right) \\
= & \int P\left(\operatorname{mes}\left(A_{\gamma}\right)<y^{d} \zeta_{1}^{-d}, M>b \mid f(\tau)=\gamma+z / b\right) h_{b}(\tau) \frac{q_{b, \tau}(\gamma+z / b)}{b} d \tau d z .
\end{aligned}
$$

The probability inside the integral is with respect to the original measure $P$ because, conditional on $f(\tau), f(t)$ follows the original conditional distribution. We develop bounds for $P\left(m e s\left(A_{\gamma}\right)<\right.$ $\left.y^{d} \zeta_{1}^{-d}, M>b \mid f(\tau)=\gamma+z / b\right)$ under two situations: $z>1$ and $0<z \leq 1$.

Situation 1: $z>1$.

There exists some constant $c_{d}>0$ only depending on the dimension $d$ such that the event $\left\{m e s\left(A_{\gamma}\right)<y^{d} \zeta_{1}^{-d}\right\}$ implies the event $\left\{\inf _{|t-\tau| \leq c_{d} y \zeta_{1}^{-1}} f(t) \leq \gamma\right\}$. Thus, we have the bound

$$
P\left(\operatorname{mes}\left(A_{\gamma}\right) \leq y^{d} \zeta_{1}^{-d}, M>b \mid f(\tau)=\gamma+\frac{z}{b}\right) \leq P\left(\inf _{|t-\tau| \leq c_{d} y \zeta_{1}^{-1}} f(t) \leq \gamma \mid f(\tau)=\gamma+\frac{z}{b}\right)
$$

Using the representation in (31), the right-hand-side of the above probability is given by

$$
=P\left(\inf _{|t| \leq c_{d} y \zeta_{1}^{-1}} f_{0}(t)+\mu_{\tau}(t) \leq \gamma\right)
$$

For $y<y_{0}$, according to Condition A2 and properties of slowly varying function, the representation (32) yields that

$$
\mu_{\tau}(t) \geq \gamma+\frac{1}{2 b} \quad \text { for }|t| \leq c_{d} y \zeta_{1}^{-1}
$$

To obtain the above bound, notice that $\mu_{\tau}(0)=\gamma+z / b>\gamma+1 / b$. In addition, for the constant variance case, expression (32) can be written as

$$
\mu_{\tau}(t)=\mu(t+\tau)+r(\tau+t, \tau)(\gamma+z / b-\mu(\tau)) .
$$

According to the continuity condition A5, we have that $\left|\mu_{\tau}(t)-\mu_{\tau}(0)\right|=O\left(b L_{1}(t)|t|^{\alpha_{1}}\right)+O\left(\sqrt{L_{t}(t)|t|^{\alpha_{1}}}\right)$. According to the definition of $\zeta_{1}$ as in (24), (36) can be achieved by choosing $y_{0}$ small. Furthermore, 
by Lemma 1(i) the conditional variance is

$$
C_{0}(t, t) \leq \lambda_{1} L_{1}\left(c_{d} y \zeta_{1}^{-1}\right) c_{d}^{\alpha_{1}} y_{1}^{\alpha} \zeta_{1}^{-\alpha_{1}}
$$

Using the slowly varying property of $L_{1}(x)$ and the fact that $L_{1}\left(\zeta_{1}^{-1}\right) \zeta_{1}^{-\alpha_{1}}=b^{-2}$, we have that

$$
L_{1}\left(c_{d} y \zeta_{1}^{-1}\right) y_{1}^{\alpha} \zeta_{1}^{-\alpha_{1}}=b^{-2} \frac{L_{1}\left(c_{d} y \zeta_{1}^{-1}\right)}{L_{1}\left(\zeta_{1}^{-1}\right)} y^{\alpha_{1}}=O\left(y^{\alpha_{1} / 2} b^{-2}\right)
$$

For the last step of the above estimate, we use Lemma 5(i) on page46 that the ratio $L_{1}\left(c_{d} y \zeta_{1}^{-1}\right) / L_{1}\left(\zeta_{1}^{-1}\right)$ varies slower than any polynomial of $y$

$$
C_{0}(t, t)=O\left(y^{\alpha_{1} / 2} b^{-2}\right)
$$

By Lemma 1(iii), $E\left(\sup _{|t| \leq c_{d} y_{0} \zeta_{1}^{-1}} b \times f_{0}(t)\right)=o(1)$ as $y_{0} \rightarrow 0$. So we can pick $y_{0}$ small enough such that

$$
E\left(\sup _{|t| \leq c_{d} y_{0} \zeta_{1}^{-1}} f_{0}(t)\right) \leq \frac{1}{4 b}
$$

By the Borel-TIS inequality (Proposition 11), (35), (36), (38), and (39), there exists a positive constant $\varepsilon_{0}$, such that

$$
P\left(\operatorname{mes}\left(A_{\gamma}\right) \leq y^{d} \zeta_{1}^{-d}, M>b \mid f(\tau)=\gamma+z / b\right) \leq P\left(\inf _{|t| \leq c_{d} y \zeta_{1}^{-1}}\left|f_{0}(t)\right|>\frac{1}{2 b}\right) \leq \exp \left(-\varepsilon_{0} y^{-\alpha_{1} / 2}\right) .
$$

Situation 2: $0<z \leq 1$.

We now proceed to the case where $0<z \leq 1$. With $y_{0}$ defined to satisfy (36) and (39), we let $c=c_{d} y_{0}$ and define a finite subset $\tilde{T}=\left\{t_{1}, \ldots, t_{N}\right\} \subset T$ such that

1. For $i \neq j, i, j \in\{1, \ldots, N\},\left|t_{i}-t_{j}\right| \geq \frac{c}{2 \zeta_{1}}$.

2. For any $t \in T$, there exists $i \in\{1, \ldots, N\}$, such that $\left|t-t_{i}\right| \leq \frac{c}{\zeta_{1}}$.

Furthermore, let

$$
B_{i}=\left\{t \in T:\left|t-t_{i}\right| \leq c \zeta_{1}^{-1}\right\} \quad \text { for } i \in\{1,2, \ldots, N\} .
$$

Thus, $\left\{B_{i}: i=1, \ldots, N\right\}$ covers $T$, that is, $\cup_{i} B_{i}=T$. Note that

$$
P\left(\operatorname{mes}\left(A_{\gamma}\right) \leq y^{d} \zeta_{1}^{-d}, M>b \mid f(\tau)=\gamma+\frac{z}{b}\right) \leq \sum_{i=1}^{N} P\left(\operatorname{mes}\left(A_{\gamma}\right) \leq y^{d} \zeta_{1}^{-d}, \sup _{t \in B_{i}} f(t)>b \mid f(\tau)=\gamma+\frac{z}{b}\right) .
$$

With $c_{d}$ as previously chosen, each of the summands in the above display is bounded by

$$
\begin{aligned}
& P\left(\operatorname{mes}\left(A_{\gamma}\right) \leq y^{d} \zeta_{1}^{-d}, \sup _{t \in B_{i}} f(t)>b \mid f(\tau)=\gamma+\frac{z}{b}\right) \\
\leq & P\left(\sup _{t \in B_{i},|s-t| \leq c_{d} y \zeta_{1}^{-1}}|f(t)-f(s)|>\frac{1}{b}, \sup _{t \in B_{i}} f(t)>b \mid f(\tau)=\gamma+\frac{z}{b}\right) .
\end{aligned}
$$

The above inequality is derived from the following argument. The process exceeds the level $b$ at some point in $B_{i}$. However, the volume of the excursion set over the level $\gamma=b-1 / b$ has to be less than $y^{d} / \zeta_{1}^{d}$. This suggests that $f(t)$ must have a fast drop from the level $b$ to $b-1 / b$. Therefore, 
the event $\left\{\operatorname{mes}\left(A_{\gamma}\right)>y^{d} \zeta_{1}^{-d}, \sup _{t \in B_{i}} f(t)>b\right\}$ is a subset of $\left\{\sup _{t \in B_{i},|s-t| \leq c_{d} y \zeta_{1}^{-1}}|f(t)-f(s)|>\right.$ $\left.\frac{1}{b}, \sup _{t \in B_{i}} f(t)>b\right\}$.

For the case that $0<z<1$, we select $\delta_{0}, \delta_{1}>0$ small enough, and $\lambda$ large enough and provide a bound for (40) under the following four cases:

Case 1. $0<\left|t_{i}-\tau\right|<y^{-\delta_{0}} \zeta_{1}^{-1}$

Case 2. $y^{-\delta_{0}} \zeta_{1}^{-1}<\left|t_{i}-\tau\right|<\delta_{1}$;

Case 3. $\left|t_{i}-\tau\right| \geq \delta_{1}, y<b^{-\lambda}$;

Case 4. $\left|t_{i}-\tau\right| \geq \delta_{1}, y \geq b^{-\lambda}$.

To facilitate the discussion, define

$$
x_{i} \triangleq \zeta_{1} \times\left|t_{i}-\tau\right|
$$

Case 1: $0<\left|t_{i}-\tau\right|<y^{-\delta_{0}} \zeta_{1}^{-1}$. We provide a bound for (40) via the conditional representation (31) and the calculation in (32). According to conditions A2 and A5, for $|t-s| \leq c_{d} y \zeta_{1}^{-1}$ and $t \in B_{i}$, we have

$$
\left|\mu_{\tau}(t)-\mu_{\tau}(s)\right| \leq \kappa_{\mu} \zeta_{1}^{-\alpha_{1} / 2} \sqrt{L_{1}\left(y / \zeta_{1}\right)} y^{\alpha_{1} / 2}+\kappa_{r}\left(x_{i}+1\right)^{\beta_{0}} L_{1}\left(\left(x_{i}+1\right) \zeta_{1}^{-1}\right) y^{\beta_{1}} \zeta_{1}^{-\alpha_{1}} b
$$

According the definition of $\zeta_{1}$ in (24), and Lemma 5 (i) the above display can be bounded by

$$
\left|\mu_{\tau}(t)-\mu_{\tau}(s)\right| \leq \frac{2 \kappa_{\mu} y^{\alpha_{1} / 4}+2 \kappa_{r} y^{-\delta_{0} \beta_{0}+\beta_{1}-\varepsilon_{0}}}{b} .
$$

We choose $\delta_{0}$ small such that it is further bounded by

$$
\left|\mu_{\tau}(t)-\mu_{\tau}(s)\right| \leq \kappa y^{\varepsilon_{0}} b^{-1} \quad \text { for some possibly different } \varepsilon_{0}>0 .
$$

Furthermore, we pick $y_{0}>0$ small enough such that for $0<y<y_{0}$ and $|s-t|<c_{d} y \zeta_{1}^{-1}$

$$
\left|\mu_{\tau}(s)-\mu_{\tau}(t)\right| \leq \frac{1}{2 b}
$$

The above inequality provides a bound on the variation of the mean function over the set $B_{i}$ when $t_{i}$ is within $y^{-\delta_{0}} \zeta_{1}^{-1}$ distance close to $\tau$. The probability in (40) can be bounded by

$$
\text { (40) } \leq P\left(\sup _{t \in B_{i},|t-s| \leq c_{d} y \zeta_{1}^{-1}}\left|f_{0}(t)-f_{0}(s)\right|>\frac{1}{2 b}\right) \text {. }
$$

Note that by Lemma 1(ii), for $|s-t|<c_{d} y \zeta_{1}^{-1}$, we have that

$$
\operatorname{Var}\left(f_{0}(s)-f_{0}(t)\right) \leq \lambda_{1} \frac{L_{1}\left(c_{d} y \zeta_{1}^{-1}\right)}{L_{1}\left(\zeta_{1}^{-1}\right)} y^{\alpha_{1}} b^{-2}=O\left(y^{\alpha_{1} / 2} b^{-2}\right) \quad \text { for } y<y_{0} .
$$


We apply the Borel-TIS inequality (Proposition 10 to the double-indexed Gaussian field $\xi(s, t) \triangleq$ $f_{0}(s)-f_{0}(t)$ and obtain that there exists a positive constant $\varepsilon_{0}$ such that

$$
\begin{aligned}
& P\left(\frac{1}{m e s\left(A_{\gamma}\right)}>y^{-d} \zeta_{1}^{d}, \sup _{t \in B_{i}} f(t)>b \mid f(\tau)=\gamma+\frac{z}{b}\right) \\
\leq & P\left(\sup _{t \in B_{i},|t-s| \leq c_{d} y \zeta_{1}^{-1}}\left|f_{0}(t)-f_{0}(s)\right|>\frac{1}{2 b}\right) \\
\leq & \exp \left(-\varepsilon_{0} y^{-\alpha_{1} / 2}\right)
\end{aligned}
$$

We put together all the $B_{i}$ 's such that $\left|t_{i}-\tau\right|<y^{-\delta_{0}} \zeta_{1}^{-1}$ and obtain that

$$
\begin{aligned}
& P\left(\frac{1}{m e s\left(A_{\gamma}\right)}>y^{-d} \zeta_{1}^{d}, \sup _{|t-\tau| \leq y^{-\delta_{0}} \zeta_{1}^{-1}} f(t)>b \mid f(\tau)=\gamma+\frac{z}{b}\right) \\
= & O\left(y^{-\delta_{0} d} \exp \left(-\varepsilon_{0} y^{-\alpha_{1} / 2}\right)\right) \leq \exp \left(-y^{-\varepsilon_{0}}\right)
\end{aligned}
$$

possibly redefining $\varepsilon_{0}$.

Case 2: $y^{-\delta_{0}} \zeta_{1}^{-1}<\left|t_{i}-\tau\right|<\delta_{1}$. For this case, we implicitly require that $y^{-\delta_{0}} \zeta_{1}^{-1}<\delta_{1}$. For $t \in B_{i}$ and $y$ small enough, we have that

$$
P\left(\sup _{t \in B_{i},|s-t| \leq c_{d} y \zeta_{1}^{-1}}|f(t)-f(s)|>\frac{1}{b}, \sup _{t \in B_{i}} f(t)>b \mid f(\tau)=\gamma+\frac{z}{b}\right) \leq P\left(\sup _{t \in B_{i}} f(t)>b \mid f(\tau)=\gamma+\frac{z}{b}\right)
$$

According to condition A2, expression (37), and property of slowly varying functions, we have the bound for $\tau+t \in B_{i}$

$$
\mu_{\tau}(t) \leq b-\frac{\Delta_{\tau}}{2} \frac{L_{1}\left(x_{i} \zeta_{1}^{-1}\right)}{L_{1}\left(\zeta_{1}^{-1}\right)} x_{i}^{\alpha_{1}} b^{-1}
$$

From Lemma 1 and definition of $\zeta_{1}$, the variance of $f_{0}(t)$ is controlled by

$$
C_{0}(t, t) \leq 2 \lambda_{1} \frac{L_{1}\left(x_{i} \zeta_{1}^{-1}\right)}{L_{1}\left(\zeta_{1}^{-1}\right)} x_{i}^{\alpha_{1}} b^{-2}
$$

According to Proposition 1 and Lemma 5 (ii) that $\frac{L_{1}\left(x_{i} \zeta_{1}^{-1}\right)}{L_{1}\left(\zeta_{1}^{-1}\right)} x_{i}^{\alpha_{1}}>x_{i}^{\alpha_{1} / 2}$ for $y^{-\delta_{0}}<x_{i}<\delta_{1} \zeta_{1}$., we continue the calculations

$$
\begin{aligned}
P\left(\sup _{t \in B_{i}} f(t)>b \mid f(\tau)=\gamma+\frac{z}{b}\right) & \leq P\left(\sup _{t+\tau \in B_{i}} f_{0}(t)>\frac{\Delta_{\tau}}{2} \frac{L_{1}\left(x_{i} \zeta_{1}^{-1}\right)}{L_{1}\left(\zeta_{1}^{-1}\right)} x_{i}^{\alpha_{1}} b^{-1}\right) \\
& \leq \exp \left(-\frac{\Delta_{\tau}^{2}}{8 \lambda_{1}} \frac{L_{1}\left(x_{i} \zeta_{1}^{-1}\right)}{L_{1}\left(\zeta_{1}^{-1}\right)} x_{i}^{\alpha_{1}}\right) \\
& \leq \exp \left(-\frac{\Delta_{\tau}^{2}}{8 \lambda_{1}} x_{i}^{\alpha_{1} / 2}\right)
\end{aligned}
$$


Putting together all the $B_{i}$ 's such that $y^{-\delta_{0}}<x_{i}<\delta_{1} \zeta_{1}$, we have that

$$
\begin{aligned}
& P\left(\frac{1}{m e s\left(A_{\gamma}\right)}>y^{-d} \zeta_{1}^{d}, \sup _{y^{-\delta_{0}} \zeta_{1}^{-1}<|t-\tau|<\delta_{1}} f(t)>b \mid f(\tau)=\gamma+\frac{z}{b}\right) \\
\leq & \sum_{k=0}^{\infty} \kappa\left(y^{-\delta_{0}}+k\right)^{d-1} \exp \left[-\frac{\Delta_{\tau}^{2}}{8 \lambda_{1}}\left(y^{-\delta_{0}}+k\right)^{\alpha_{1} / 2}\right] \\
\leq & \exp \left(-y^{-\varepsilon_{0}}\right)
\end{aligned}
$$

for some constant $\varepsilon_{0}>0$.

Case 3: $\left|t_{i}-\tau\right| \geq \delta_{1}$ and $y<b^{-\lambda}$. Since $C(s, t)$ is uniformly Hölder continuous, we can always choose $\lambda$ large such that for $|s-t| \leq c_{d} y \zeta_{1}^{-1} \leq c_{d} b^{-\lambda} \zeta_{1}^{-1}$,

$$
\left|\mu_{\tau}(t)-\mu_{\tau}(s)\right| \leq \frac{1}{4 b}
$$

By Lemma 1(ii) and Lemma 5 (i), for $|s-t| \leq c_{d} y \zeta_{1}^{-1}$, the conditional variance $\operatorname{Var}\left(f_{0}(s)-f_{0}(t)\right)$ is bounded by

$$
\operatorname{Var}\left(f_{0}(s)-f_{0}(t)\right) \leq \lambda_{1} \frac{L_{1}\left(c_{d} y \zeta_{1}^{-1}\right)}{L_{1}\left(\zeta_{1}^{-1}\right)} y^{\alpha_{1}} b^{-2}=O\left(y^{\alpha_{1} / 2} b^{-2}\right) .
$$

Thus, there exist a constant $\varepsilon_{0}>0$ such that

$$
\begin{aligned}
& P\left(\sup _{t \in B_{i},|s-t| \leq c_{d} y \zeta_{1}^{-1}}|f(t)-f(s)|>\frac{1}{b}, \sup _{t \in B_{i}} f(t)>b \mid f(\tau)=\gamma+\frac{z}{b}\right) \\
\leq & P\left(\sup _{t \in B_{i},|s-t| \leq c_{d} y \zeta_{1}^{-1}}\left|f_{0}(t)-f_{0}(s)\right|>\frac{1}{2 b}\right) \\
\leq & 2 \exp \left(-\varepsilon_{0} y^{-\alpha_{1}}\right)
\end{aligned}
$$

Note that $\zeta_{1} \ll b^{4 / \alpha_{1}}$, so for $y<b^{-\lambda}$, we have

$$
\begin{aligned}
& P\left(\frac{1}{m e s\left(A_{\gamma}\right)}>y^{-d} \zeta_{1}^{d}, \sup _{|t-\tau|>\delta_{1}} f(t)>b \mid f(\tau)=\gamma+\frac{z}{b}\right) \\
\leq & O\left(\zeta_{1}^{d}\right) \sup _{i} P\left(\sup _{t \in B_{i},|s-t| \leq c_{d} y \zeta_{1}^{-1}}|f(t)-f(s)|>\frac{1}{b}, \sup _{t \in B_{i}} f(t)>b \mid f(\tau)=\gamma+\frac{z}{b}\right) \\
\leq & O\left(b^{4 d / \alpha_{1}}\right) \exp \left(-\varepsilon_{0} y^{-\alpha_{1} / 2}\right) \\
\leq & O\left(y^{-\frac{4 d}{\alpha_{1} \lambda}}\right) \exp \left(-\varepsilon_{0} y^{-\alpha_{1} / 2}\right) \\
\leq & \exp \left(-y^{-\varepsilon_{0}}\right)
\end{aligned}
$$

for some possibly different constant $\varepsilon_{0}$.

Case 4: $\left|t_{i}-\tau\right| \geq \delta_{1}$ and $y \geq b^{-\lambda}$. Note that condition A3 implies that for any $\delta_{1}>0$, there exists $\varepsilon>0$ such that for $|s-t|>\delta_{1}, r(s, t)<1-\varepsilon$, and thus according to expression (37), there exists $\varepsilon>0$ such that $\mu_{\tau}(t) \leq(1-\varepsilon) b$. According to Proposition 1 (the Borel-TIS inequality), we 
have that for $b$ large enough,

$$
\begin{aligned}
& P\left(\frac{1}{m e s\left(A_{\gamma}\right)}>y^{-d} \zeta_{1}^{d}, \sup _{|t-\tau| \geq \delta_{1}} f(t)>b \mid f(\tau)=\gamma+\frac{z}{b}\right) \\
\leq & P\left(\sup _{|t| \geq \delta_{1}} f_{0}(t)+\mu_{\tau}(t)>b\right) \\
\leq & P\left(\sup _{|t| \geq \delta_{1}} f_{0}(t)>\varepsilon b\right) \\
\leq & \exp \left(-\frac{\varepsilon^{2} b^{2}}{2 \sigma_{T}^{2}}\right) \\
\leq & \exp \left(-y^{-\varepsilon_{0}}\right)
\end{aligned}
$$

for some constant $\varepsilon_{0}>0$.

Combining Cases 1-4, for some constants $\varepsilon_{0}$ and $y_{0}$ chosen to be small, we have that for $y \in\left(0, y_{0}\right]$

$$
P\left(\frac{1}{m e s\left(A_{\gamma}\right)}>y^{-d} \zeta_{1}^{d}, M>b \mid f(\tau)=\gamma+\frac{z}{b}\right) \leq \exp \left(-y^{-\varepsilon_{0}}\right)
$$

Together with (34), we have

$$
Q\left(\frac{1}{m e s\left(A_{\gamma}\right)}>y^{-d} \zeta_{1}^{d}, M>b\right) \leq \exp \left(-y^{-\varepsilon_{0}}\right)
$$

Thus, according to (33), for some $\kappa>0$, we have

$$
E^{Q}\left[\frac{1}{m e s\left(A_{\gamma}\right)^{2}} ; M>b\right] \leq\left(\kappa+y_{0}^{-2 d}\right) \zeta_{1}^{2 d}
$$

\subsection{The $I_{2}$ term}

To provide a lower bound of

$$
I_{2}=E^{Q}\left[\frac{1}{m e s\left(A_{\gamma}\right)} ; M>b\right]
$$

we basically need to prove that mes $\left(A_{\gamma}\right)$ cannot be always very large. Thus, it is sufficient to show that $f(t)$ drop below $\gamma$ when $t$ is reasonably far away from $\tau$. The next lemma shows that for any $\delta>0$, the process $f(t)$ drops below $\gamma$ almost all the time when $|t-\tau|>\delta$.

Lemma 2 Under conditions A1-6, for standard deviation of Type 1, we have that

$$
Q\left(\sup _{|t-\tau|>\delta} f(t) \geq \gamma\right) \leq e^{-\varepsilon_{0} b^{2}} \quad \text { for some } \varepsilon_{0}>0 .
$$

Lemma 3 Under conditions A1-6, there exists $\delta$ small and $\kappa$ large (independent of $b$ ), such that for $x>\kappa$ we have

$$
Q\left(\sup _{x \zeta^{-1} \leq|t-\tau| \leq \delta} f(t) \geq \gamma\right)<e^{-\varepsilon_{0} x^{\alpha_{1} / 4}}
$$

The proof of these two Lemmas are provided in the Supplemental Material. We proceed to developing a lower bound for $I_{2}$. First, notice that the event $\{M>b\}$ is a regular event under $Q$, that is,

$$
Q(M>b) \geq Q(f(\tau)>b)>\frac{1}{2} e^{-1 / \sigma^{2}(\tau)} .
$$


Also, according to Lemma 2 and 3, we choose $x$ such that

$$
Q\left(\sup _{|t-\tau|>x \zeta_{1}^{-1}} f(t) \geq \gamma\right)<\frac{1}{2} e^{-2 / \sigma^{2}(\tau)}
$$

Let $\omega_{d}$ be the volume of the $d$-dimensional unit ball. Thus, we have

$$
\begin{aligned}
I_{2} & \geq E^{Q}\left(\frac{1}{\operatorname{mes}\left(A_{\gamma}\right)} ; M>b, \operatorname{mes}\left(A_{\gamma}\right)<\omega_{d} x^{d} \zeta_{1}^{-d}\right) \\
& \geq \omega_{d}^{-1} x^{-d} \zeta_{1}^{d} Q\left(\operatorname{mes}\left(A_{\gamma}\right)<\omega_{d} x^{d} \zeta_{1}^{-d}, M>b\right) \\
& \geq \omega_{d}^{-1} x^{-d} \zeta_{1}^{d}\left[Q(M>b)-Q\left(\operatorname{mes}\left(A_{\gamma}\right) \geq \omega_{d} x^{d} \zeta_{1}^{-d}\right)\right] \\
& \geq \omega_{d}^{-1} x^{-d} \zeta_{1}^{d}\left[Q(M>b)-Q\left(\sup _{|t-\tau|>x \zeta_{1}^{-1}} f(t) \geq \gamma\right)\right] \\
& \geq \omega_{d}^{-1} x^{-d} \zeta_{1}^{d}\left(e^{-1 / \sigma^{2}(\tau)}-e^{-2 / \sigma^{2}(\tau)}\right)
\end{aligned}
$$

Summarizing the results in (50) and (53), we have that

$$
E_{b}\left(Z_{b}^{2}\right) \leq \kappa \zeta_{1}^{2 d}\left(\int P(f(t)>\gamma) d t\right)^{2}, \quad P(M>b)>\varepsilon_{0} \zeta_{1}^{d} \int P(f(t)>\gamma) d t
$$

and therefore

$$
\sup _{b} \frac{E^{Q} Z_{b}^{2}}{P^{2}(M>b)}<\infty
$$

\section{Proof of Theorem 2}

Let $T_{m}=\left\{t_{1}, \ldots, t_{m}\right\}$ be generated in the step 3 of Algorithm 2. We start the analysis with the following decomposition

$$
\begin{aligned}
\hat{Z}_{b}-Z_{b}= & {\left[\frac{I(\sup f(t)>b)}{\operatorname{mes}\left(A_{\gamma}\right)}-\frac{I\left(\max _{i=1}^{m} f\left(t_{i}\right)>b\right)}{\widehat{m e s}\left(A_{\gamma}\right)}\right] E\left(\operatorname{mes}\left(A_{\gamma}\right)\right) } \\
= & E\left(\operatorname{mes}\left(A_{\gamma}\right)\right) \\
& \quad \times\left[\frac{I(\sup f(t)>b)}{\operatorname{mes}\left(A_{\gamma}\right)}-\frac{I\left(\max _{i=1}^{m} f\left(t_{i}\right)>b\right)}{\operatorname{mes}\left(A_{\gamma}\right)}\right. \\
& \left.\quad+\frac{I\left(\max _{i=1}^{m} f\left(t_{i}\right)>b\right)}{\operatorname{mes}\left(A_{\gamma}\right)}-\frac{I\left(\max _{i=1}^{m} f\left(t_{i}\right)>b\right)}{\widehat{m e s}\left(A_{\gamma}\right)}\right],
\end{aligned}
$$

where $\widehat{m e s}\left(A_{\gamma}\right)$ is defined as in (26). According to the result in Theorem 1, we only need to show that $\left|E^{Q}\left(\hat{Z}_{b}-Z_{b}\right)\right| \leq \varepsilon P(M>b)$ and $\operatorname{Var}\left(\hat{Z}_{b}-Z_{b}\right)=O\left(P^{2}(M>b)\right)$. We define notations

$$
\begin{aligned}
J_{1} & =\frac{I(\sup f(t)>b)}{\operatorname{mes}\left(A_{\gamma}\right)}-\frac{I\left(\max _{i=1}^{m} f\left(t_{i}\right)>b\right)}{\operatorname{mes}\left(A_{\gamma}\right)} \\
J_{2} & =\frac{I\left(\max _{i=1}^{m} f\left(t_{i}\right)>b\right)}{\operatorname{mes}\left(A_{\gamma}\right)}-\frac{I\left(\max _{i=1}^{m} f\left(t_{i}\right)>b\right)}{\widehat{m e s}\left(A_{\gamma}\right)} .
\end{aligned}
$$

We control each of the two terms respectively. 


\subsection{The $J_{1}$ term}

Note that $J_{1}$ is non-negative and

$$
E_{b}\left(J_{1}\right)=E_{b}\left(\frac{1}{\operatorname{mes}\left(A_{\gamma}\right)} ; M>b ; \max _{i=1}^{m} f\left(t_{i}\right) \leq b\right) .
$$

Note that the proof of Theorem 1, in particular (49) and (81), shows that $\frac{I(M>b)}{\zeta^{d} m e s\left(A_{\gamma}\right)}$ is uniformly integrable in the parameter $b$ where

$$
\zeta=\max \left(\zeta_{1}, \zeta_{2}\right)
$$

More precisely, for any $\delta$ small enough, we have that

$$
\sup _{Q(B) \leq \delta} E_{b}\left(\frac{1}{m e s\left(A_{\gamma}\right)} ; M>b ; B\right) \leq(-\log \delta)^{1 / \varepsilon_{0}} \delta \zeta^{d} .
$$

Therefore, it is sufficient to focus on and derive a bound for the probaiblity

$$
Q\left(M>b ; \max _{i=1}^{m} f\left(t_{i}\right) \leq b\right) .
$$

Let $x$ be large and $\delta^{\prime}$ be small such that

$$
\begin{aligned}
& Q\left(M>b ; \max _{i=1}^{m} f\left(t_{i}\right) \leq b\right) \\
\leq & Q\left(\sup _{x \zeta^{-1}<|t-\tau|<\delta^{\prime}} f(t)>b ; \max _{i=1}^{m} f\left(t_{i}\right) \leq b\right) \\
+ & Q\left(\sup _{|t-\tau|<x \zeta^{-1}} f(t)>b, \sup _{|t-\tau|>x \zeta^{-1}} f(t) \leq b ; \max _{i=1}^{m} f\left(t_{i}\right) \leq b\right) \\
+ & Q\left(\sup _{|t-\tau| \geq \delta^{\prime}} f(t)>b ; \max _{i=1}^{m} f\left(t_{i}\right) \leq b\right) .
\end{aligned}
$$

We will provide a specific choice of $m$ such that

$$
Q\left(\sup f(t)>b ; \max _{i=1}^{m} f\left(t_{i}\right) \leq b\right) \leq \delta \triangleq \varepsilon^{1+\varepsilon_{0}},
$$

where $\varepsilon$ is relative bias preset in the statement of the theorem. We consider each of the three terms in (56).

\subsubsection{The first term in (56).}

We choose

$$
x=\min \left\{(-\log \delta)^{4 / \alpha}, \delta^{\prime} \zeta\right\}, \quad \text { where } \alpha=\min \left\{\alpha_{1}, \alpha_{2}\right\} .
$$

According to Lemma 3 and (83), the first term in (56) is bounded by

$$
Q\left(\sup _{x \zeta^{-1}<|t-\tau|<\delta^{\prime}} f(t)>b ; \max _{i=1}^{m} f\left(t_{i}\right) \leq b\right) \leq Q\left(\sup _{x \zeta^{-1}<|t-\tau|<\delta^{\prime}} f(t)>b\right) \leq \delta .
$$

Notationally, we define that $\sup _{t \in \emptyset} f(t)=-\infty$. Thus, when $x=\delta^{\prime} \zeta$, the above probability is zero. 


\subsubsection{The second term in (56).}

Simple derivations yield that

$$
\begin{aligned}
& Q\left(\sup _{|t-\tau|<x \zeta^{-1}} f(t)>b, \sup _{|t-\tau|>x \zeta^{-1}} f(t) \leq b, \max _{i=1}^{m} f\left(t_{i}\right) \leq b\right) \\
= & E_{b}\left[Q\left(\max _{i=1}^{m} f\left(t_{i}\right) \leq b \mid f\right) ; \sup _{|t-\tau|<x \zeta^{-1}} f(t)>b, \sup _{|t-\tau|>x \zeta^{-1}} f(t) \leq b\right] \\
\leq & E_{b}\left[\left(1-\beta\left(A_{b}\right)\right)^{m} ; \sup _{|t-\tau|<x \zeta^{-1}} f(t)>b\right]
\end{aligned}
$$

where

$$
\beta\left(A_{b}\right)=\zeta^{d} \times \operatorname{mes}\left(A_{b} \cap B(\tau, x / \zeta)\right) \times \inf _{|t| \leq x} k(t)
$$

is the lower bound of the probability that $Q\left(t_{i} \in A_{b} \mid f\right)$ and $B(\tau, x)$ is the ball centered around $\tau$ with radius $x$. In what follows, we need to show that mes $\left(A_{b}\right)$ cannot be too small on the set $\left\{\sup _{|t-\tau|<x \zeta^{-1}} f(t)>b\right\}$ and therefore $\beta\left(A_{b}\right)$ cannot be too small. We write

$$
\mathcal{E}_{1}=\left\{\sup _{|t-\tau|<x \zeta^{-1}} f(t)>b\right\}
$$

and write (57) as

$$
E_{b}\left[\left(1-\beta\left(A_{b}\right)\right)^{m} ; \mathcal{E}_{1}\right]=E_{b}\left[\left(1-\beta\left(A_{b}\right)\right)^{m} ; \mathcal{E}_{1}, D_{\lambda_{3}, \delta_{1}}^{c}\right]+E_{b}\left[\left(1-\beta\left(A_{b}\right)\right)^{m} ; \mathcal{E}_{1}, D_{\lambda_{3}, \delta_{1}}\right]
$$

where, for some $\lambda_{3}$ and $\delta_{1}$ positive, we define

$$
D_{\lambda_{3}, \delta_{1}}=\left\{\sup _{\substack{|s-t| \leq \lambda_{3} \zeta^{-1} \\ s, t \in B\left(\tau, x \zeta^{-1}\right)}}|f(s)-f(t)| \leq \delta_{1} b^{-1}\right\} .
$$

For some $\varepsilon_{0}$ small, we choose $\delta_{1}=\varepsilon_{0} \delta$ and

$$
\lambda_{3}=\varepsilon_{0} \delta_{1}^{2 / \alpha+1 / \beta_{1}+\varepsilon_{0}} .
$$

We apply the Borel-TIS lemma to the double-indexed process $\xi(s, t)=f(s)-f(t)$ whose variance is bounded by Lemma1(ii). Thus, we obtain the following bound

$$
E_{b}\left[\left(1-\beta\left(A_{b}\right)\right)^{m} ; \mathcal{E}_{1}, D_{\lambda_{3}, \delta_{1}}^{c}\right] \leq Q\left(D_{\lambda_{3}, \delta_{1}}^{c}\right) \leq \delta .
$$

Therefore, (57) is bounded by

$$
\delta+E_{b}\left[\left(1-\beta\left(A_{b}\right)\right)^{m} ; \mathcal{E}_{1}, D_{\lambda_{3}, \delta_{1}}\right] .
$$

We further split the expectation

$$
\begin{aligned}
E_{b}\left[\left(1-\beta\left(A_{b}\right)\right)^{m} ; \mathcal{E}_{1}, D_{\lambda_{3}, \delta_{1}}\right] \leq & E_{b}\left[\left(1-\beta\left(A_{b}\right)\right)^{m} ; D_{\lambda_{3}, \delta_{1}} ; \sup _{|t-\tau|<x \zeta^{-1}} f(t)>b+\delta_{1} b^{-1}, \mathcal{E}_{1}\right] \\
& +Q\left[D_{\lambda_{3}, \delta_{1}} ; b<\sup _{|t-\tau|<x \zeta^{-1}} f(t) \leq b+\delta_{1} b^{-1}, \mathcal{E}_{1}\right] .
\end{aligned}
$$


We proceed to providing a bound of the second term by considering the standardized process $g(t)=b(f(\tau+t / \zeta)-b)$ conditional on $f(\tau)=\gamma+\frac{z}{b} \cdot g(t)$ can be written as

$$
g(t)=\frac{C(t / \zeta+\tau)}{C(\tau, \tau)} z+l(t)
$$

where $l(t)$ is a random field whose distribution is independent of $z$. So we have

$$
\begin{aligned}
Q\left(b<\sup _{|t-\tau|<x \zeta^{-1}} f(t)<b+\delta_{1} b^{-1}\right) & =Q\left(\sup _{|t| \leq x} \frac{C(t / \zeta+\tau)}{C(\tau, \tau)} z+l(t) \in\left(0, \delta_{1}\right)\right) \\
& =O\left(\delta_{1}\right)
\end{aligned}
$$

The last equality holds because $z$ has a density bounded everywhere (asymptotically exponential), and $\frac{1}{2}<\frac{C(t / \zeta+\tau)}{C(\tau, \tau)}<\frac{\sigma_{T}^{2}}{\sigma^{2}(\tau)}$. Given a realization of $l(t), \sup _{|t| \leq x} \frac{C(t / \zeta+\tau)}{C(\tau, \tau)} z+l(t) \in\left(0, \delta_{1}\right)$ implies that $z$ has to fall in an interval with length less than $2 \delta_{1}$. Thus, if we choose $\varepsilon_{0}$ small and $\delta_{1}=\varepsilon_{0} \delta$, then

$$
Q\left(b<\sup _{|t-\tau|<x \zeta^{-1}} f(t)<b+\delta_{1} \zeta^{-1}\right)<\delta .
$$

Therefore, we have that (57) is bounded by

$$
2 \delta+E^{Q}\left[\left(1-\beta\left(A_{b}\right)\right)^{m} ; D_{\lambda_{3}, \delta_{1}} ; \sup _{|t-\tau|<x \zeta^{-1}} f(t)>b+\delta_{1} b^{-1}, \mathcal{E}_{1}\right] .
$$

Note that, on the set $D_{\lambda_{3}, \delta_{1}}$, mes $\left(A_{b} \cap B\left(\tau, x \zeta^{-1}\right)\right)$ is controlled by the overshoot $\sup _{|t-\tau|<x \zeta^{-1}} f(t)-$ $b$, that is, if $\sup _{|t-\tau|<x \zeta^{-1}} f(t)>b+\delta_{1} / b$, then $\operatorname{mes}\left(A_{b} \cap B\left(\tau, x \zeta^{-1}\right)\right) \geq \varepsilon_{0} \lambda_{3}^{d} \zeta^{-d}$. In addition, the density $k_{\tau, \zeta}(t)$ is bounded from below by $x^{-d-\varepsilon_{1}}$ for $t \in B\left(\tau, x \zeta^{-1}\right)$. Thus, the probability $\beta\left(A_{b}\right)$ has a lower bound

$$
\beta\left(A_{b}\right) \geq \varepsilon_{0} x^{-d-\varepsilon_{1}} \lambda_{3}^{d} \geq \varepsilon_{0} \delta^{2 d / \alpha+d / \beta_{1}+2 \varepsilon_{0}} .
$$

The last step of the above inequality follows from that $x=\min \left\{(-\log \delta)^{4 / \alpha}, \delta^{\prime} \zeta\right\}$. Thus, we have that (57) is bounded by

$$
2 \delta+\left(1-\varepsilon_{0} \delta^{2 d / \alpha+d / \beta_{1}+2 \varepsilon_{0}}\right)^{m} .
$$

We choose for some $\varepsilon_{0}$ and $\kappa>0$

$$
m=\kappa \delta^{-2 d / \alpha-d / \beta_{1}-2 \varepsilon_{0}}
$$

and therefore

$$
Q\left(\sup _{|t-\tau|<x \zeta^{-1}} f(t)>b, \sup _{|t-\tau|>x \zeta^{-1}} f(t) \leq b ; \max _{i=1}^{m} f\left(t_{i}\right) \leq b\right) \leq 4 \delta
$$

\subsubsection{The last term in (56).}

According to the result in Lemma 2 (and the corresponding result as in (82) for the non-constant variance case presented in the Supplemental Material), we can choose $\varepsilon_{0}$ and $\delta^{\prime}$ such that

$$
Q\left(\sup _{|t-\tau| \geq \delta^{\prime}} f(t) \geq \gamma\right) \leq e^{-\varepsilon_{0} b^{2}} .
$$

There are two cases: $\delta>e^{-\varepsilon_{0} b^{2}}$ and $\delta \leq e^{-\varepsilon_{0} b^{2}}$. 
Case 1: $\delta>e^{-\varepsilon_{0} b^{2}}$. In this case, The last term in (156) is bounded trivially by

$$
Q\left(\sup _{|t-\tau| \geq \delta^{\prime}} f(t)>b ; \max _{i=1}^{m} f\left(t_{i}\right) \leq b\right) \leq Q\left(\sup _{|t-\tau| \geq \delta^{\prime}} f(t) \geq \gamma\right) \leq \delta
$$

Case 2: $\delta<e^{-\varepsilon_{0} b^{2}}$. We need a similar analysis to that of the second term. We now split the probability for $\delta_{2}=\delta^{1+\varepsilon_{0}}$

$$
\begin{aligned}
& Q\left(\sup _{|t-\tau| \geq \delta^{\prime}} f(t)>b ; \max _{i=1}^{m} f\left(t_{i}\right) \leq b\right) \\
& \quad \leq Q\left(\sup _{|t-\tau| \geq \delta^{\prime}} f(t) \in\left[b, \delta_{2} b^{-\lambda}\right]\right)+Q\left(\sup _{|t-\tau| \geq \delta^{\prime}} f(t)>b+\delta_{2} b^{-\lambda} ; \max _{i=1}^{m} f\left(t_{i}\right) \leq b\right) .
\end{aligned}
$$

We now consider the first term split the set $\left\{t:|t-\tau|>\delta^{\prime}\right\}$ into two parts. Define the set $F=\left\{t: \frac{C(t, \tau)}{C(\tau, \tau)}>\frac{1}{\left(-\log \delta_{2}\right)^{2}}\right\}$, We start with the small overshoot probability on the set $F$

$$
Q\left(b<\sup _{|t-\tau|>\delta^{\prime}, t \in F} f(t) \leq b+\delta_{2} / b\right)
$$

Using the representation (58), applying similar analysis as that of the second term, we have that

$$
\begin{aligned}
& Q\left(b<\sup _{|t-\tau| \geq \delta^{\prime}, t \in F} f(t)<b+\delta_{2} b^{-1}\right) \\
& \quad \leq Q\left(\sup _{\left|\frac{t}{\zeta}\right|>\delta^{\prime}, \frac{t}{\zeta}+\tau \in F} \frac{C(t / \zeta+\tau)}{C(\tau, \tau)} z+l(t) \in\left(0, \delta_{2}\right)\right)=O\left(\left(-\log \delta_{2}\right)^{2} \delta_{2}\right) \leq \delta .
\end{aligned}
$$

The last two steps are based on the fact that $z$ is a random variable independent of $l(t)$ and has bounded density. Thus, the above probability is bounded by

$$
\sup _{x} P\left(x<z<x+\left(\log \delta_{2}\right)^{2} \delta_{2}\right)=O\left(\left(\log \delta_{2}\right)^{2} \delta_{2}\right) .
$$

We will return to this estimate soon.

We now consider $t$ in $F^{c}$. For some $\kappa_{0}$ large, we have that $Q\left(z>-\kappa_{0} \log \delta_{2}\right)<\delta_{2}$. Thus, we only consider $z<-\kappa_{0} \log \delta_{2}$. Conditional on $f(\tau)=\gamma+z / b$, conditional mean is $\sup _{t \in F^{c}} \mu_{\tau}(t-\tau) \leq$ $C>0$. In addition, the conditional variance of $f(t)$ on the set $F^{c}$ is almost $\sigma^{2}(t)$. Thus, we can apply classic results on the density estimation of the sup $f(t)$ (c.f. Theorem 2 of [32]) and have that conditional on $f(\tau)=\gamma+\frac{z}{b}$, $\sup _{|t-\tau| \geq \delta^{\prime}, F^{c}} f(t)$ has a bounded density over $\left[b, b+\delta_{2} b^{-\lambda}\right]$ for some $\lambda \geq 1$, and thus

$$
Q\left(\sup _{|t-\tau| \geq \delta^{\prime}, t \in F^{c}} f(t) \in\left[b, b+\delta_{2} b^{-\lambda}\right] \mid f(\tau)=\gamma+\frac{z}{b}\right)=O\left(\delta_{2}\right)
$$


Summarizing the above results, we have that

$$
\begin{aligned}
& Q\left(\sup _{|t-\tau| \geq \delta^{\prime}} f(t) \in\left[b, b+\delta_{2} b^{-\lambda}\right]\right) \\
\leq & Q\left(\sup _{|t-\tau| \geq \delta^{\prime}, t \in F} f(t) \in\left[b, b+\delta_{2} b^{-\lambda}\right]\right) \\
& +Q\left(z \geq-\kappa_{0} \log \delta_{2}\right)+Q\left(\sup _{|t-\tau| \geq \delta^{\prime}, t \in F^{c}} f(t) \in\left[b, b+\delta_{2} b^{-\lambda}\right], z \leq-\kappa_{0} \log \delta_{2}\right) \\
\leq & 3 \delta .
\end{aligned}
$$

Thus, the last term in (56) is bounded by

$$
\begin{aligned}
& Q\left(\sup _{|t-\tau| \geq \delta^{\prime}} f(t)>b ; \max _{i=1}^{m} f\left(t_{i}\right) \leq b\right) \\
& \quad \leq 3 \delta+Q\left(\sup _{|t-\tau| \geq \delta^{\prime}} f(t)>b+\delta_{2} b^{-\lambda} ; \max _{i=1}^{m} f\left(t_{i}\right) \leq b\right) .
\end{aligned}
$$

For the second term, we apply the old trick by choosing

$$
\lambda_{4}=\delta_{2}^{2 / \alpha+1 / \beta_{1}+\varepsilon_{0}} b^{-2 \lambda / \alpha-\lambda / \beta_{1}}
$$

and thus

$$
Q\left(\sup _{|s-t|<\lambda_{4}}|f(s)-f(t)|>\delta_{2} b^{-\lambda}\right)<\delta_{2}
$$

Note that $b^{2} \leq-\varepsilon_{0}^{-1} \log \delta_{2}$, we can choose a different $\varepsilon_{0}$ such that $\lambda_{4}$ can be simplified to

$$
\lambda_{4}=\delta_{2}^{2 / \alpha+1 / \beta_{1}+\varepsilon_{0}} .
$$

If $\sup _{|s-t|<\lambda_{4}}|f(s)-f(t)|<\delta_{2} b^{-\lambda}$ and $\sup _{|t-\tau| \geq \delta^{\prime}} f(t)>b+\delta_{2} b^{-\lambda}$, we have that

$$
\beta\left(A_{b}\right) \geq \varepsilon_{0} \lambda_{4}^{d} \zeta^{-d-\varepsilon_{1}} .
$$

With a different choice of $\varepsilon_{0}$, we choose

$$
m=-2 \lambda_{4}^{-d} \zeta^{d+\varepsilon_{1}} \log \delta=O\left(\delta^{-d\left(2 / \alpha+1 / \beta_{1}\right)-\varepsilon_{0}}\right),
$$

then we have

$$
E_{b}\left[\left(1-\beta\left(A_{b}\right)\right)^{m} ; \sup _{|s-t|<\lambda_{4}} b<|f(s)-f(t)|<\delta_{2} b^{-\lambda}, f(t)>b+\delta_{2} b^{-\lambda}\right] \leq \delta
$$

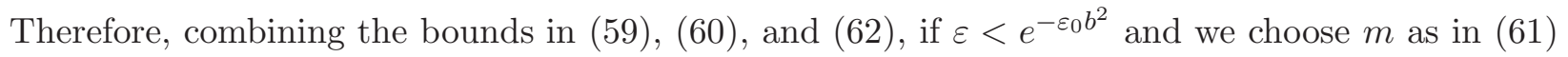
and, then

$$
Q\left(\sup _{|t-\tau|>\delta^{\prime}} f(t)>b ; \max _{i=1}^{m} f\left(t_{i}\right) \leq b\right) \leq 5 \delta
$$

Putting together the bounds for all the three terms in (56), we have that

$$
Q\left(M>b ; \max _{i=1}^{m} f\left(t_{i}\right) \leq b\right) \leq 5 \delta .
$$


If we choose $\delta=\varepsilon^{1+\varepsilon_{0}}$ and

$$
m=O\left(\delta^{-d\left(2 / \alpha+1 / \beta_{1}+\varepsilon_{0}\right)}\right)=O\left(\varepsilon^{-d\left(2 / \alpha+1 / \beta_{1}\right)-2 d \varepsilon_{0}}\right)
$$

then according to the bounds on the bound in (55), we have that

$$
E^{Q} J_{1} \leq \zeta^{d} \varepsilon
$$

Similarly, according to the integrability of $\zeta^{-2 d} / \mathrm{mes}^{2}\left(A_{\gamma}\right)$, by choosing the same $m$, there exists a $\kappa_{0}$ such that

$$
E^{Q}\left(J_{1}^{2}\right) \leq \kappa_{0} \zeta^{2 d}
$$

\subsection{The $J_{2}$ term}

We now proceed to

$$
J_{2}=I\left(\max _{i=1}^{m} f\left(t_{i}\right)>b\right)\left[\frac{1}{\operatorname{mes}\left(A_{\gamma}\right)}-\frac{1}{\widehat{m e s}\left(A_{\gamma}\right)}\right] .
$$

We study the behavior of $J_{2}$ by means of the scaled process $g(t)$ defined as in (22). For the analysis of $J_{2}$, we translate everything to the scale of $g(t)$. Recall the process $g(t)$ given by (22) is

$$
g(t)=b(f(\tau+t / \zeta)-b)
$$

For each $t, f(\tau+t / \zeta)>\gamma$ if and only if $g(t)>-1$.

Conditional on $\tau, t_{1}, \ldots, t_{m}$ are i.i.d. with density $k_{\tau, \zeta}(t)$ defined as in (25). Let $s_{i}=\left(t_{i}-\tau\right) \zeta$ and thus $s_{1}, \ldots, s_{m}$ are i.i.d. following density $k(s)$. We can then rewrite the estimator in (26) as

$$
\widehat{m e s}\left(A_{\gamma}\right)=\frac{\zeta^{-d}}{m} \sum_{i=1}^{m} \frac{I\left(g\left(s_{i}\right)>-1\right)}{k\left(s_{i}\right)} .
$$

Thus, $\widehat{m e s}\left(A_{\gamma}\right)$ is an unbiased estimator of $m e s\left(A_{\gamma}\right)$, that is, $E\left(\widehat{m e s}\left(A_{\gamma}\right) \mid f\right)=m e s\left(A_{\gamma}\right)$. Conditional on a particular realization of $f(t)$ (or equivalently, $\mathrm{g}(\mathrm{t})$ ), the variance of $\widehat{m e s}\left(A_{\gamma}\right)$ is given by

$$
\operatorname{Var}\left(\widehat{m e s}\left(A_{\gamma}\right) \mid f\right)=\frac{\kappa_{f} \zeta^{-2 d}}{m}
$$

where

$$
\kappa_{f}=\operatorname{Var}\left[\frac{I(g(S)>-1)}{k(S)} \mid f\right] \leq k^{-2}\left(t_{f}\right)
$$

and

$$
t_{f}=\max (|t|: g(t)>-1) .
$$

Note that the following inequality $\frac{1}{1+x}-1 \geq-x$. Therefore,

$$
\frac{1}{m e s\left(A_{\gamma}\right)}-\frac{1}{\widehat{m e s}\left(A_{\gamma}\right)} \leq \frac{\widehat{m e s}\left(A_{\gamma}\right)-m e s\left(A_{\gamma}\right)}{m e s^{2}\left(A_{\gamma}\right)}
$$

Therefore,

$$
E\left[\left(\frac{1}{m e s\left(A_{\gamma}\right)}-\frac{1}{\widehat{m e s}\left(A_{\gamma}\right)}\right)^{2} ; \widehat{m e s}\left(A_{\gamma}\right)>\operatorname{mes}\left(A_{\gamma}\right) \mid f\right] \leq \frac{\kappa_{f} \zeta^{-2 d}}{m \times m e s^{4}\left(A_{\gamma}\right)} .
$$


It is the expectation on the set $\left\{\widehat{m e s}\left(A_{\gamma}\right)<\operatorname{mes}\left(A_{\gamma}\right)\right\}$ that induces complications in that the factor $\frac{1}{\overline{m e s}\left(A_{\gamma}\right)}$ can be very large when there are not many $t_{i}$ 's in the excursion set $A_{\gamma}$. We now proceed to this case. Conditional on a particular realization of $f$ (and equivalently the process $g(t)$ ), the analysis consists of three steps.

Step 1. Define the $f$-dependent probability

$$
p_{f} \triangleq Q\left(t_{i} \in A_{\gamma} \mid f\right)=\int_{A_{\gamma}} k_{\tau, \zeta}(t) d t=\int_{A_{-1}^{g}} k(t) d t
$$

Using standard exponential change of measure techniques for large deviations [14, we obtain that

$$
Q\left[\sum_{i=1}^{m} I\left(t_{i} \in A_{\gamma}\right) \leq p_{f}\left(1-\delta_{3}\right) m \mid f\right] \leq e^{-m I\left(\delta_{3}, p_{f}\right)}
$$

for all $\delta_{3} \in(0,1)$, where the rate function

$$
I\left(\delta_{3}, p_{f}\right)=\theta_{*} p_{f}\left(1-\delta_{3}\right)-\varphi\left(\theta_{*}\right)
$$

and

$$
\varphi(\theta)=\log \left(1-p_{f}+p_{f} e^{\theta}\right), \quad \theta_{*}=\log \left(1-\frac{\delta_{3}}{1-p_{f}\left(1-\delta_{3}\right)}\right) .
$$

By elementary calculus, if we choose $\delta_{3}=\frac{1}{2}$, then we have that for some $\varepsilon_{0}>0$

$$
I\left(\delta_{3}, p_{f}\right) \geq \varepsilon_{0} p_{f} \quad \text { for all } p_{f}>0 .
$$

Therefore,

$$
\begin{aligned}
& E\left[\left(\frac{1}{m e s\left(A_{\gamma}\right)}-\frac{1}{\widehat{m e s}\left(A_{\gamma}\right)}\right)^{2} ; \widehat{m e s}\left(A_{\gamma}\right) \leq \operatorname{mes}\left(A_{\gamma}\right), \max _{i=1}^{m} f\left(t_{i}\right)>b, \sum_{i=1}^{m} I\left(t_{i} \in A_{\gamma}\right) \leq \frac{p_{f} m}{2} \mid f\right] \\
\leq & E\left[\frac{4}{\widehat{m e s}^{2}\left(A_{\gamma}\right)} ; \widehat{m e s}\left(A_{\gamma}\right) \leq \operatorname{mes}\left(A_{\gamma}\right), \max _{i=1}^{m} f\left(t_{i}\right)>b, \sum_{i=1}^{m} I\left(t_{i} \in A_{\gamma}\right) \leq \frac{p_{f} m}{2} \mid f\right] .
\end{aligned}
$$

Note that there is at least one $t_{i}$ in the excursion set $A_{\gamma}$. Therefore, the estimator $\widehat{m e s}\left(A_{\gamma}\right) \geq$ $m^{-1} \zeta^{-d} k^{-1}\left(t_{f}\right)$. Thus, the above expectation is upper bounded by

$$
\leq \kappa k\left(t_{f}\right)^{-2} m^{2} \zeta^{2 d} e^{-\varepsilon_{0} m p_{f}} .
$$

Step 2. We consider the situation that $\sum I\left(t_{i} \in A_{\gamma}\right)>\frac{p_{f} m}{2}$. The unbiasedness of $\widehat{m e s}\left(A_{\gamma}\right)$ suggests that

$$
\operatorname{mes}\left(A_{\gamma}\right)=E\left(\frac{1}{\zeta^{d} k(S)} \mid S \in A_{-1}^{g}\right) p_{f},
$$

where $S$ is a random index following density $k(s)$. Note that on the set $A_{-1}^{g}, k\left(t_{f}\right) \leq k(S) \leq \kappa_{1}$. Thus, if we let $\lambda_{f}=\kappa_{1}^{-1} k\left(t_{f}\right)$, then on the set $\left\{\sum I\left(t_{i} \in A_{\gamma}\right)>\frac{p_{f} m}{2}\right\}$ we have

$$
\widehat{m e s}\left(A_{\gamma}\right) \geq \frac{\lambda_{f} m e s\left(A_{\gamma}\right)}{2} \text {. }
$$


Thus, using Taylor expansion, we have that

$$
\begin{aligned}
& E_{b}\left[\left(\frac{1}{m e s\left(A_{\gamma}\right)}-\frac{1}{\widehat{m e s}\left(A_{\gamma}\right)}\right)^{2} ; \widehat{m e s}\left(A_{\gamma}\right)<\operatorname{mes}\left(A_{\gamma}\right) ; \sum I\left(t_{i} \in A_{\gamma}\right)>\frac{p_{f} m}{2} \mid f\right] \\
\leq & E_{b}\left[\frac{2^{4}\left(m e s\left(A_{\gamma}\right)-\widehat{m e s}\left(A_{\gamma}\right)\right)^{2}}{\lambda_{f}^{4} m e s^{4}\left(A_{\gamma}\right)} ; \widehat{m e s}\left(A_{\gamma}\right)<\operatorname{mes}\left(A_{\gamma}\right) ; \sum I\left(t_{i} \in A_{\gamma}\right)>\frac{p_{f} m}{2} \mid f\right] \\
\leq & \frac{2^{4} \kappa_{f} \zeta^{-2 d}}{m \lambda_{f}^{4} m e s^{4}\left(A_{\gamma}\right)} .
\end{aligned}
$$

Step 3. We combine the previous analysis and have that

$$
E_{b}\left(J_{2}^{2} \mid f\right) \leq \frac{2^{4} \zeta^{-2 d}}{m e s^{4}\left(A_{\gamma}\right)} \frac{\kappa_{1}^{4}}{k^{4}\left(t_{f}\right) m}+\frac{\kappa_{f} \zeta^{-2 d}}{m \times m e s^{4}\left(A_{\gamma}\right)}+k\left(t_{f}\right)^{-2} m^{2} \zeta^{2 d} e^{-\varepsilon_{0} m p_{f}} .
$$

The density $k(t)$ has a heavy tail that is

$$
k(t) \sim \frac{1}{|t|^{d+\varepsilon_{1}}}
$$

and $k(t) \leq \kappa_{1}$ for all $t$. In Step 3, we provide a bound on the distribution of $t_{f}$ and $p_{f}$.

We start with $t_{f}$. For each $s>0, t_{f}>s$ if and only if $\sup _{|t-\tau|>s} g(t)>-1$. According to the results in Lemmas 2 and 3 (and the corresponding bounds in (82) and (83) for the non-constant variance case presented in the Supplemental Material), for $s$ sufficiently large, there exists some $\varepsilon_{0}>0$ such that

$$
Q\left(t_{f}>s\right)=Q\left(\sup _{|t-\tau|>s} g(t)>-1\right) \leq \exp \left\{-s^{\varepsilon_{0}}\right\}, \quad \text { for } s<\delta^{\prime} \zeta
$$

and

$$
Q\left(t_{f}>s\right) \leq \exp \left(-\varepsilon_{0} b^{2}\right), \quad \text { for } s>\delta^{\prime} \zeta .
$$

Therefore, all moments of $k^{-1}\left(t_{f}\right)$ is bounded.

$$
E_{b}\left[k^{-l}\left(t_{f}\right)\right] \leq E_{b}\left[t_{f}^{\left(d+\epsilon_{1}\right) l}\right] \leq \kappa_{l}
$$

for some constant $\kappa_{l}$ possibly depending on $l$. Thus, by Cauchy-Schwarz inequality, the expectation of the first two terms in (68) can be bounded as follows

$$
\begin{aligned}
E\left[\frac{2^{4} \zeta^{-2 d}}{m e s^{4}\left(A_{\gamma}\right)} \frac{\kappa_{1}^{4}}{k^{4}\left(t_{f}\right) m} ; M>b\right] & \leq \frac{O(1)}{m} \sqrt{E\left[\frac{\zeta^{-4 d}}{m e s^{8}\left(A_{\gamma}\right)}\right] E\left(k^{-8}\left(t_{f}\right)\right)} \leq \frac{\kappa \zeta^{2}}{m} \\
E\left[\frac{\kappa_{f} \zeta^{-2 d}}{m \times m e s^{4}\left(A_{\gamma}\right)}\right] & \leq \frac{O(1)}{m} \sqrt{E\left[\frac{\zeta^{-4 d}}{m e s^{8}\left(A_{\gamma}\right)}\right] E\left(k^{-4}\left(t_{f}\right)\right)} \leq \frac{\kappa \zeta^{2}}{m} .
\end{aligned}
$$

We now proceed to the third term in (68) concerning $p_{f}$. The expectation of this term is bounded by

$$
E_{b}\left(m^{2} k\left(t_{f}\right)^{-2} e^{-m \varepsilon_{0} p_{f}} ; M>b\right) \leq \sqrt{E_{b}\left(m^{4} e^{-2 m \varepsilon_{0} p_{f}} ; M>b\right)} \sqrt{E_{b}\left(k^{-4}\left(t_{f}\right)\right)} .
$$


The second term $\sqrt{E_{b}\left(k^{-4}\left(t_{f}\right)\right)}$ is $O(1)$. We proceed to the first term

$$
\begin{aligned}
E_{b}\left(m^{4} e^{-2 m \varepsilon_{0} p_{f}} ; M>b\right) & =E_{b}\left(m^{4} e^{-2 m \varepsilon_{0} p_{f}} ; p_{f} \geq m^{-1 / 2}\right)+E_{b}\left(m^{4} e^{-2 m \varepsilon_{0} p_{f}} ; p_{f} \leq m^{-1 / 2}, M>b\right) \\
& \leq m^{4} e^{-2 \varepsilon_{0} \sqrt{m}}+m^{4} Q\left(p_{f} \leq m^{-1 / 2}, M>b\right) .
\end{aligned}
$$

We now proceeding to controlling $Q\left(p_{f} \leq m^{-1 / 2}, M>b\right)$. Note that

$$
p_{f} \geq k\left(t_{f}\right) \operatorname{mes}\left(A_{-1}^{g}\right) .
$$

For each $x>0$,

$$
\begin{aligned}
Q\left(p_{f}<x, M>b\right) & \leq Q\left(k\left(t_{f}\right)<\sqrt{x} \text { or } \operatorname{mes}\left(A_{-1}^{g}\right)<\sqrt{x}, M>b\right) \\
& \leq Q\left(t_{f}>x^{-\frac{1}{2\left(d+\varepsilon_{1}\right)}}\right)+Q\left(\operatorname{mes}\left(A_{-1}^{g}\right)<\sqrt{x}, M>b\right)
\end{aligned}
$$

According to the bounds in (48) and (81), for some $\delta_{0}>0$ and $\varepsilon_{0}>0$, we have that

$$
Q\left(\operatorname{mes}\left(A_{-1}^{g}\right)<\sqrt{x}, M>b\right)=Q\left(\operatorname{mes}\left(A_{\gamma}\right)<\zeta^{-d} \sqrt{x}, M>b\right) \leq e^{-x^{-\varepsilon_{0}} / d}
$$

for $x$ sufficiently small. According to the previous result, we have that

$$
Q\left(t_{f}>x^{-\frac{1}{2\left(d+\varepsilon_{1}\right)}}\right) \leq e^{-x^{-\varepsilon_{0}}}, \quad \text { for } x^{-\frac{1}{2\left(d+\varepsilon_{1}\right)}}<\delta^{\prime} \zeta
$$

and

$$
Q\left(t_{f}>x^{-\frac{1}{2\left(d+\varepsilon_{1}\right)}}\right) \leq e^{-\varepsilon_{0} b^{2}}, \text { for } x^{-\frac{1}{2\left(d+\varepsilon_{1}\right)}} \geq \delta^{\prime} \zeta
$$

Thus, for some $\lambda$ large enough and $\varepsilon_{0}$ small enough, we have that

$$
Q\left(p_{f} \leq m^{-1 / 2}, M>b\right) \leq e^{-m^{\varepsilon_{0}}}, \quad \text { for } m<b^{\lambda}
$$

for $m>b^{\lambda}$ (with $\lambda$ sufficiently large), $t_{f}>m^{\frac{1}{4\left(d+\varepsilon_{1}\right)}}$ implies that $\tau+t_{f} / \zeta \notin T$, that is, $m^{\frac{1}{4\left(d+\varepsilon_{1}\right)}}$ is too large and thus

$$
Q\left(p_{f}<m^{-1 / 2}\right)=0, \quad \text { for } m>b^{\lambda} .
$$

Therefore,

$$
m^{4} Q\left(p_{f} \leq m^{-1 / 2}, M>b\right) \leq \kappa m^{4} e^{-m^{\varepsilon_{0}}}
$$

for $m$ sufficiently large and furthermore

$$
E_{b}\left(m^{4} k\left(t_{f}\right)^{-2} e^{-m \varepsilon_{0} p_{f}} ; M>b\right) \leq \kappa m^{4} e^{-m^{\varepsilon_{0}} / 2} .
$$

Summarizing the results in all the three steps, we have that

$$
E_{b}\left(J_{2}^{2}\right) \leq \frac{\kappa \zeta^{-2 d}}{m}
$$

Therefore, if we choose

$$
m=\kappa \max \left\{\varepsilon^{-2}, \varepsilon^{-d\left(2 / \alpha+1 / \beta_{1}+\varepsilon_{0}\right)}\right\}=O\left(\varepsilon^{-d\left(2 / \alpha+2 / \beta_{1}\right)}\right)
$$


then,

$$
E_{b}\left|\hat{Z}_{b}-Z_{b}\right|=E_{b}\left|J_{1}+J_{2}\right| \int_{T} P(f(t)>\gamma) d t \leq \varepsilon \zeta^{d} \int_{T} P(f(t)>\gamma) d t
$$

and

$$
E_{b}\left(\hat{Z}_{b}-Z_{b}\right)^{2} \leq \kappa \zeta^{2 d}\left(\int_{T} P(f(t)>\gamma) d t\right)^{2}
$$

\section{Proof of Theorem 3}

\subsection{The asymptotic lower bound and the continuous estimator}

We start the analysis by first establishing an asymptotic lower bound of $v(b)$. Note that

$$
v(b)=E\left(\operatorname{mes}\left(A_{\gamma}\right)\right) E_{b}\left[\frac{1}{m e s\left(A_{\gamma}\right)} \int_{A_{b}} \xi(t) d t\right] .
$$

Since $\xi(t)$ is bounded by $a_{2}$, then $v(b) \leq a_{2} E\left(\right.$ mes $\left.\left(A_{\gamma}\right)\right)$. In addition, a lower bound can be given by

$$
E\left(\int_{A_{b}} \xi(t) d t\right) \geq a_{1} E\left(\operatorname{mes}\left(A_{b}\right)\right)
$$

Thus

$$
v(b)=\Theta(1) E\left(\operatorname{mes}\left(A_{\gamma}\right)\right)
$$

The second moment of the estimator is

$$
E_{b}\left(Y_{b}^{2}\right)=E^{2}\left(\operatorname{mes}\left(A_{\gamma}\right)\right) E_{b}\left[\frac{\alpha^{2}(b)}{\operatorname{mes}^{2}\left(A_{\gamma}\right)} ; M>b\right] \leq a_{2}^{2} E^{2}\left(\operatorname{mes}\left(A_{\gamma}\right)\right) \leq \frac{a_{2}^{2}}{a_{1}^{2}} v(b) .
$$

\subsection{Analysis of the discrete estimator}

We start the analysis by the following decomposition

$$
\begin{aligned}
\hat{Y}_{b}-Y_{b}= & {\left[\frac{\alpha(b)}{\operatorname{mes}\left(A_{\gamma}\right)} I(\sup f(t)>b)-\frac{\hat{\alpha}(b)}{\widehat{m e s}\left(A_{\gamma}\right)} I\left(\max _{i=1}^{m} f\left(t_{i}\right)>b\right)\right] E\left(\operatorname{mes}\left(A_{\gamma}\right)\right) } \\
= & E\left(\operatorname{mes}\left(A_{\gamma}\right)\right) \\
& \times\left[\frac{\alpha(b) I(\sup f(t)>b)}{\operatorname{mes}\left(A_{\gamma}\right)}-\frac{\alpha(b) I\left(\max _{i=1}^{m} f\left(t_{i}\right)>b\right)}{\operatorname{mes}\left(A_{\gamma}\right)}\right. \\
& \left.+\frac{\alpha(b) I\left(\max _{i=1}^{m} f\left(t_{i}\right)>b\right)}{\operatorname{mes}\left(A_{\gamma}\right)}-\frac{\hat{\alpha}(b) I\left(\max _{i=1}^{m} f\left(t_{i}\right)>b\right)}{\widehat{m e s}\left(A_{\gamma}\right)}\right] .
\end{aligned}
$$

We redefine the terms

$$
\begin{aligned}
& J_{1}=\frac{\alpha(b) I(\sup f(t)>b)}{\operatorname{mes}\left(A_{\gamma}\right)}-\frac{\alpha(b) I\left(\max _{i=1}^{m} f\left(t_{i}\right)>b\right)}{\operatorname{mes}\left(A_{\gamma}\right)} \\
& J_{2}=\frac{\alpha(b) I\left(\max _{i=1}^{m} f\left(t_{i}\right)>b\right)}{\operatorname{mes}\left(A_{\gamma}\right)}-\frac{\hat{\alpha}(b) I\left(\max _{i=1}^{m} f\left(t_{i}\right)>b\right)}{\widehat{\operatorname{mes}}\left(A_{\gamma}\right)} .
\end{aligned}
$$


Note that the factor $\alpha(b) /$ mes $\left(A_{\gamma}\right)$ is bounded by $a_{2}$, so we have

$$
E_{b}\left|J_{1}\right| \leq a_{2} Q\left(\sup f(t)>b, \max _{i=1}^{m} f\left(t_{i}\right)>b\right), \quad E_{b}\left(J_{1}^{2}\right) \leq a_{2}^{2} Q\left(\sup f(t)>b, \max _{i=1}^{m} f\left(t_{i}\right)>b\right)
$$

According to the previous analysis, for each $\varepsilon$, there exists an $m=O\left(\varepsilon^{\left.-d\left(2 / \alpha+1 / \beta_{1}\right)-\varepsilon_{0}\right)}\right.$ such that

$$
E_{b}\left(\left|J_{1}\right| \quad \mid f\right) \leq a_{2} \varepsilon, \quad E_{b}\left(J_{1}^{2} \mid f\right)=a_{2}^{2} \varepsilon .
$$

For the second term, we apply similar analysis as the proof for Theorem 2 . Note that $\alpha(b) \leq$ $a_{2} m e s\left(A_{\gamma}\right)$, so by rearranging terms in $J_{2}$, we have

$$
\left|J_{2}\right| \leq\left[\frac{|\alpha(b)-\hat{\alpha}(b)|}{m e s\left(A_{\gamma}\right)}+a_{2} \frac{\left|m e s\left(A_{\gamma}\right)-\widehat{m e s}\left(A_{\gamma}\right)\right|}{m e s\left(A_{\gamma}\right)}\right] I(M>b) .
$$

Because $\hat{\alpha}(b)$ is an unbiased estimator for $\alpha(b)$ conditional on $f$, we have

$$
E_{b}\left[(\hat{\alpha}(b)-\alpha(b))^{2} \mid f\right] \leq m^{-1} a_{2}^{2} k^{-2}\left(t_{f}\right) \zeta^{-2 d} .
$$

Thus,

$$
\begin{aligned}
& E_{b}\left[(\hat{a}(b)-\alpha(b))^{2}+a_{2}\left(\operatorname{mes}\left(A_{\gamma}\right)-\widehat{\operatorname{mes}}\left(A_{\gamma}\right)\right)^{2} \mid f\right] \\
\leq & 2 E_{b}\left[(\hat{\alpha}(b)-\alpha(b))^{2} \mid f\right]+2 a_{2}^{2} E_{b}\left[\left(\operatorname{mes}\left(A_{\gamma}\right)-\widehat{m e s}\left(A_{\gamma}\right)\right) \mid f\right] \\
\leq & 4 a_{2}^{2} m^{-1} k^{-2}\left(t_{f}\right) \zeta^{-2 d} .
\end{aligned}
$$

Therefore,

$$
\begin{aligned}
E_{b}\left(\left|J_{2}\right|^{2} \mid f\right) & \leq \frac{4 a_{2}^{2}}{\lambda_{f}^{2} m e s\left(A_{\gamma}\right)^{2} \zeta^{2 d} m k^{2}\left(t_{f}\right)} \\
E_{b}\left(\left|J_{2}\right| \mid f\right) & \leq \frac{2 a_{2}}{\lambda_{f} m e s\left(A_{\gamma}\right) \zeta^{d} \sqrt{m} k\left(t_{f}\right)}
\end{aligned}
$$

According the proof in Section 6, there exists a $\kappa>0$ such that

$$
E\left(\left|J_{2}\right|\right) \leq \frac{\kappa}{\sqrt{m}}
$$

With a similar argument, we have that

$$
E\left(J_{2}^{2}\right) \leq \kappa
$$

Summarizing the result for $J_{1}$ and $J_{2}$, we can choose $m=O\left(\max \left(\varepsilon^{-d\left(2 / \alpha+1 / \beta_{1}+\varepsilon_{0}\right)}, \varepsilon^{-2}\right)\right)=$ $O\left(\varepsilon^{-d\left(2 / \alpha+2 / \beta_{1}\right)}\right)$, such that

$$
E_{b}\left(\hat{Y}_{b}-v(b)\right) \leq \varepsilon v(b), \quad \operatorname{Var}\left(\hat{Y}_{b}\right)=O(1) .
$$




\section{References}

[1] R.J. Adler. The Geometry of Random Fields. Wiley, Chichester, U.K.; New York, U.S.A., 1981.

[2] R.J. Adler, J.H. Blanchet, and J. Liu. Efficient monte carlo for high excursions of gaussian random fields. The Annals of Applied Probability, 22(3):1167-1214, 2012.

[3] R.J. Adler, G. Samorodnitsky, and J.E. Taylor. High level excursion set geometry for nonGaussian infinitely divisible random fields. preprint, 2009.

[4] R.J. Adler and J.E. Taylor. Random fields and geometry. Springer, 2007.

[5] S. Asmussen and P. Glynn. Stochastic Simulation: Algorithms and Analysis. Springer, New York, NY, USA, 2007.

[6] J. M. Azais and M. Wschebor. A general expression for the distribution of the maximum of a Gaussian field and the approximation of the tail. Stochastic Processes and Their Applications, 118(7):1190-1218, 2008.

[7] J. M. Azais and M. Wschebor. Level sets and extrema of random processes and fields. Wiley, Hoboken, N.J., 2009.

[8] S. M. Berman. An asymptotic formula for the distribution of the maximum of a Gaussian process with stationary increments. Journal of Applied Probability, 22(2):454-460, 1985.

[9] N.H. Bingham, C.M. Goldie, and J.L. Teugels. Regular variation, volume 27. Cambridge university press, 1989.

[10] C. Borell. The Brunn-Minkowski inequality in Gauss space. Inventiones Mathematicae, 1975.

[11] C. Borell. The Brunn-Minkowski inequality in Gauss space. Invent. Math., 30:205-216, 1975.

[12] C. Borell. The Ehrhard inequality. Comptes Rendus Mathematique, 337(10):663-666, 2003.

[13] J. Bucklew. Introduction to Rare Event Simulation. Springer, New York, NY, USA, 2004.

[14] A. Dembo and O. Zeitouni. Large deviations techniques and applications, volume 38. Springer, 2009 .

[15] R.M. Dudley. Sample functions of the gaussian process. Selected Works of RM Dudley, pages 187-224, 2010.

[16] S. Juneja and P. Shahabuddin. Rare event simulation techniques: An introduction and recent advances. Handbook on Simulation, pages 291-350, 2006.

[17] H. J. Landau and L. A. Shepp. Supremum of a Gaussian process. Sankhya-the Indian Journal of Statistics Series A, 32(Dec):369-378, 1970.

[18] M. Ledoux and M. Talagrand. Probability in Banach spaces : isoperimetry and processes. 1991.

[19] J. Liu. Tail approximations of integrals of Gaussian random fields. Annals of Probability, to appear, 2011. 
[20] J. Liu and G. Xu. On the conditional distributions and the efficient simulations of exponential integrals of gaussian random fields. Arxiv preprint arXiv:1204.5546, 2012.

[21] J. Liu and G. Xu. Some asymptotic results of Gaussian random fields with varying mean functions and the associated processes. Annals of Statistics, Accepted.

[22] M. B. Marcus and L. A. Shepp. Continuity of Gaussian processes. Transactions of the American Mathematical Society, 151(2), 1970.

[23] M. Mitzenmacher and E. Upfal. Probability and Computing: Randomized Algorithms and Probabilistic Analysis. Cambridge University Press, 2005.

[24] V. I. Piterbarg. Asymptotic methods in the theory of Gaussian processes and fields. American Mathematical Society, Providence, R.I., 1996.

[25] V.N. Sudakov and B.S. Tsirelson. Extremal properties of half spaces for spherically invariant measures. Zap. Nauchn. Sem. LOMI, 45:75-82, 1974.

[26] J. Y. Sun. Tail probabilities of the maxima of Gaussian random-fields. Annals of Probability, 21(1):34-71, 1993.

[27] M. Talagrand. Majorizing measures: The generic chaining. Annals of Probability, 24(3):10491103, 1996.

[28] J. Taylor, A. Takemura, and R. J. Adler. Validity of the expected Euler characteristic heuristic. Annals of Probability, 33(4):1362-1396, 2005.

[29] J.E. Taylor and R.J. Adler. Euler characteristics for Gaussian fields on manifolds. Annals of Probability, 31(2):533-563, 2003.

[30] J. Traub, G. Wasilokowski, and H. Wozniakowski. Information-Based Complexity. Academic Press, New York, NY, 1988.

[31] B.S. Tsirelson, I.A. Ibragimov, and V.N. Sudakov. Norms of Gaussian sample functions. Proceedings of the Third Japan-USSR Symposium on Probability Theory (Tashkent, 1975), 550:20-41, 1976.

[32] V. S. Tsirel'son. The density of the maximum of a gaussian process. Theory of Probab. Appl., 20:847-856, 1975.

[33] H. Wozniakowski. Computational complexity of continuous problems, 1996. Technical Report. 


\section{Supplemental Material}

\section{A Proof of Theorem 1 when $\sigma(t)$ is of Type 2 in Assumption A4}

In our proof for Type 2 standard deviation, we use similar methods as that for Type 1 . We are going to establish similar results as in (48) and Lemmas 2 and 3 hold for Gaussian random field with type 2 standard deviation. To proceed, we provide some bounds on the distribution of $\tau$. The next lemma suggests that $\tau$ is close to

$$
t^{*}=\arg \sup _{t \in T} \sigma(t)
$$

Lemma 4 There exists constants $\delta, \varepsilon_{0}>0$ small enough and $\kappa>0$ large enough (but independent of b), such that for $x>\kappa$ the following bounds hold

(i) $\int_{\left|t-t^{*}\right| \leq \zeta_{2}^{-1}} h_{b}(t) d t>\varepsilon_{0}$,

(ii) $\int_{\delta>\left|t-t^{*}\right|>x \zeta_{2}^{-1}} h_{b}(t) d t<\exp \left(-x^{\alpha_{2} / 2}\right)$,

(iii) $\int_{\left|t-t^{*}\right|>\delta} h_{b}(t) d t<\exp \left(-\varepsilon_{0} b^{2}\right)$.

To continue the analysis of $I_{1}$ and $I_{2}$, we discuss two different scenarios:

1. $\alpha_{1}>\alpha_{2}$, or $\alpha_{1}=\alpha_{2}$ and $\lim _{x \rightarrow 0} \frac{L_{1}(x)}{L_{2}(x)} \in\{0,1\}$; that is, as $x \rightarrow 0, L_{1}(x) x^{\alpha_{1}} \leq(1+$ $o(1)) L_{2}(x) x^{\alpha_{2}}$.

2. $\alpha_{1}<\alpha_{2}$, or $\alpha_{1}=\alpha_{2}$ and $\lim _{x \rightarrow 0} \frac{L_{1}(x)}{L_{2}(x)}=\infty$; that is, as $x \rightarrow 0, L_{2}(x) x^{\alpha_{2}}=o(1) L_{1}(x) x^{\alpha_{1}}$.

The proof of this lemma is provided in the Supplemental Material B.

A.1 Proof for scenario 1: $\alpha_{1}>\alpha_{2}$, or $\alpha_{1}=\alpha_{2}$ and $\lim _{x \rightarrow 0} \frac{L_{1}(x)}{L_{2}(x)} \in\{0,1\}$.

For the proof of this scenario, the variation of $\sigma(t)$ is the dominating term. According to A2, there exists a constant $\Delta$ such that

$$
1-r(s, t) \leq \Delta L_{2}(|s-t|)|s-t|^{\alpha_{2}}
$$

In addition, we can further replace the slowly varying function $L_{1}$ in (8) by $L_{2}$ and the inequality still holds, that is,

$$
\left|r\left(t, t+s_{1}\right)-r\left(t, t+s_{2}\right)\right| \leq \kappa_{r} \max \left(L_{2}\left(\left|s_{1}\right|\right)\left|s_{1}\right|^{\beta_{0}}, L_{2}\left(\left|s_{2}\right|\right)\left|s_{2}\right|^{\beta_{0}}\right)\left|s_{1}-s_{2}\right|^{\beta_{1}} .
$$

For the proof of this scenario, we work under the above two inequalities instead of A2. The proof follows a similar idea as that of the constant variance case by providing bounds for $I_{1}$ and $I_{2}$.

The $I_{1}$ term. For a given $\tau$ and $z$, we adopt a similar conditional representation as in (31). We start with establishing similar results as in Lemma 1. Since $L_{1}(x) x^{\alpha_{1}} \leq(1+o(1)) L_{2}(x) x^{\alpha_{2}}$, we can replace $\alpha_{1}$ and $L_{1}$ in the statement of Lemma 1 by $\alpha_{2}$ and $L_{2}$ and the statement still holds. Now 
we proceed to prove (48). According to the expression (34), we proceed by deriving an upper bound of

$$
\int_{T} P\left(\frac{1}{\operatorname{mes}\left(A_{\gamma}\right)}>y^{-d} \zeta_{2}^{-1}, M>b \mid f(\tau)=\gamma+\frac{z}{b}\right) h_{b}(\tau) \frac{q_{b, \tau}(\gamma+z / b)}{b} d \tau d z . \quad \text { for } y \text { small enough. }
$$

We discuss two situation: $z>1$ and $0<z \leq 1$.

Situation 1: $z>1$. From condition A2, A4, A5, (72) and Lemma 5 (i), for $|t|<c_{d} y \zeta_{2}^{-1}$, we have that

$$
\left|\mu_{\tau}(t)-\mu_{\tau}(0)\right| \leq \kappa_{\mu} \sqrt{L_{2}(|t|)}|t|^{\alpha_{2} / 2}+\kappa b L_{2}(|t|)|t|^{\alpha_{2}}=O\left(y^{\alpha_{2} / 4} b^{-1}\right)
$$

Note that $\mu_{\tau}(0)=\gamma+z / b>\gamma+1 / b$. Thus, by picking $y_{0}$ small enough, we have that

$$
\mu_{\tau}(t) \geq \gamma+\frac{1}{2 b} \quad \text { for }|t| \leq c_{d} y \zeta_{2}^{-1}
$$

With a similar development as in (35) and the conditional variance calculation for $f_{0}(t)$ as in (38), that is,

$$
C_{0}(t, t)=O\left(y^{\alpha_{2} / 2} b^{-2}\right),
$$

we conclude that for some small $\varepsilon_{0}>0$

$Q\left(\frac{1}{m e s\left(A_{\gamma}\right)}>y^{-d} \zeta_{2}^{-1}, M>b\right) \leq P\left(\inf _{|t| \leq c_{d} y \zeta_{2}^{-1}}\left(f_{0}(t)+\mu_{\tau}(t)\right) \leq \gamma\right) \leq P\left(\inf _{|t| \leq c_{d} y \zeta_{2}^{-1}}\left|f_{0}(t)\right|>\frac{1}{2 b}\right) \leq \exp \left(-y^{-\varepsilon_{0}}\right)$.

Situation 2: $0<z \leq 1$. For $0<z<1$, we choose $\delta_{0}, \delta_{1}$ to be small enough and $\lambda$ to be large enough and develop bounds for the above probability under four cases (same as in the proof of constant variance case):

Case 1. $t \in C_{1} \triangleq\left\{t: 0<|t-\tau|<y^{-\delta_{0}} \zeta_{2}^{-1}\right\}$,

Case 2. $t \in C_{2} \triangleq\left\{t: y^{-\delta_{0}} \zeta_{2}^{-1}<|t-\tau|<\delta_{1}\right\}$,

Case 3. $t \in C_{3} \triangleq\left\{t:|t-\tau| \geq \delta_{1}\right\}$ and $y<b^{-\lambda}$,

Case 4. $t \in C_{3}$ and $y \geq b^{-\lambda}$.

With these notation, we have the following bound

$$
\begin{aligned}
& Q\left(\frac{1}{\operatorname{mes}\left(A_{\gamma}\right)}>y^{-d} \zeta_{2}^{-1}, M>b\right) \\
& \quad \leq \sum_{i=1}^{3} \int_{T} P\left(\frac{1}{m e s\left(A_{\gamma}\right)}>y^{-d} \zeta^{d}, \sup _{t \in C_{i}} f(t)>b \mid f(\tau)=\gamma+\frac{z}{b}\right) h_{b}(\tau) \frac{q_{b, \tau}(\gamma+z / b)}{b} d \tau d z .
\end{aligned}
$$


With the same argument for (40), each of the summands on the right-hand-side is further bounded by

$$
\begin{aligned}
\int_{T} P & \left(\frac{1}{\operatorname{mes}\left(A_{\gamma}\right)}>y^{-d} \zeta_{2}^{d}, \sup _{t \in C_{i}} f(t)>b \mid f(\tau)=\gamma+\frac{z}{b}\right) h_{b}(\tau) \frac{q_{b, \tau}(\gamma+z / b)}{b} d \tau d z \\
& \leq \int_{T} P\left(\sup _{t \in C_{i},|s-t| \leq c_{d} y \zeta_{2}^{-1}}|f(t)-f(s)|>\frac{1}{b}, \sup _{t \in C_{i}} f(t)>b \mid f(\tau)=\gamma+\frac{z}{b}\right) h_{b}(\tau) \frac{q_{b, \tau}(\gamma+z / b)}{b} d \tau d z
\end{aligned}
$$

Similarly, we define

$$
x_{i} \triangleq \zeta_{2} \times\left|t_{i}-\tau\right|
$$

Case 1: $0<|t-\tau|<y^{-\delta_{0}} \zeta_{2}^{-1}$. We adopt the same lattice and cover sets, $\tilde{T}$, and $B_{i}$, defined on page 19 for the proof of the constant variance case, with $\zeta_{1}$ replaced by $\zeta_{2}$. For this case, we bound the right-hand-side of (74) by

$$
\sum_{B_{i} \cap C_{1} \neq \emptyset} \int_{T} P\left(\sup _{t \in B_{i},|s-t| \leq c_{d} y \zeta_{2}^{-1}}|f(t)-f(s)|>\frac{1}{b} \mid f(\tau)=\gamma+\frac{z}{b}\right) h_{b}(\tau) \frac{q_{b, \tau}(\gamma+z / b)}{b} d \tau d z
$$

and take advantage of the conditional representation $f(t)=\mu_{\tau}(t)+f_{0}(t)$. We proceed to investigating the variation of $\mu_{\tau}(t)$ and $f_{0}(t)$. For $f_{0}(t)$ and $|s-t| \leq c_{d} y \zeta_{2}^{-1}$, with the same argument as in (42), we have that $\operatorname{Var}\left(f_{0}(t)-f_{0}(s)\right) \leq \kappa y^{\alpha_{2} / 2} b^{-2}$. For the conditional mean, by means of the representation (32),

$$
\begin{aligned}
\left|\mu_{\tau}(t)-\mu_{\tau}(s)\right| & \leq \kappa \zeta_{2}^{-\alpha_{2} / 2} \sqrt{L_{2}\left(y / \zeta_{2}\right)} y^{\alpha_{2} / 2}+\kappa b L_{2}\left(c_{d} y / \zeta_{2}\right) y^{\alpha_{2}} \zeta_{2}^{-\alpha_{2}}+\kappa\left(x_{i}+1\right)^{\beta_{0}} b L_{2}\left(\left(x_{i}+1\right) / \zeta_{2}\right) y^{\beta_{1}} \zeta_{2}^{-\alpha_{2}} \\
& \leq \kappa b^{-1} y^{\varepsilon_{0}}
\end{aligned}
$$

for some small positive constant $\varepsilon_{0}$. Now we pick $y_{0}$ small enough. For $0<y<y_{0}$ and $\mid \mu_{\tau}(t)-$ $\mu_{\tau}(s) \mid<\frac{1}{2 b}$, together with the variance control of $f_{0}(t)-f_{0}(s)$, we have that

$$
\begin{aligned}
& \int_{T} P\left(\sup _{t \in B_{i},|s-t| \leq c_{d} y \zeta_{2}^{-1}}|f(t)-f(s)|>\frac{1}{b} \mid f(\tau)=\gamma+\frac{z}{b}\right) h_{b}(\tau) d \tau \\
& \quad \leq \int_{T} P\left(\sup _{t \in B_{i},|s-t| \leq c_{d} y \zeta_{2}^{-1}}\left|f_{0}(t)-f_{0}(s)\right|>\frac{1}{2 b} \mid f(\tau)=\gamma+\frac{z}{b}\right) h_{b}(\tau) d \tau \\
& \quad \leq \exp \left(-y^{-\varepsilon_{0}}\right)
\end{aligned}
$$

for some $\varepsilon_{0}>0$. We sum up all the $B_{i}$ 's such that $0<\left|t_{i}-\tau\right|<y^{-\delta_{0}} \zeta_{2}^{-1}$ and obtain that

$$
P\left(\frac{1}{m e s\left(A_{\gamma}\right)}>y^{-d} \zeta_{1}^{d}, \sup _{t \in C_{1}} f(t)>b \mid f(\tau)=\gamma+\frac{z}{b}\right) \leq \exp \left(-y^{-\varepsilon_{0}}\right)
$$

for which we may need to choose a smaller $\varepsilon_{0}$. 
Case 2: $y^{-\delta_{0}} \zeta_{2}^{-1}<|t-\tau|<\delta_{1}$. We split (74) as follows

$$
\begin{aligned}
(74) \leq & \sum_{B_{i} \cap C_{2} \neq \emptyset} \int_{\left|\tau-t^{*}\right| \leq \frac{1}{3} y^{-\delta_{0}} \zeta_{2}^{-1}} P\left(\sup _{t \in B_{i}} f(t)>b \mid f(\tau)=\gamma+\frac{z}{b}\right) h_{b}(\tau) d \tau \\
& +\int_{\left|\tau-t^{*}\right|>\frac{1}{3} y^{-\delta_{0}} \zeta_{2}^{-1}} h_{b}(\tau) d \tau .
\end{aligned}
$$

For this case, we implicitly requires that $y^{-\delta_{0}}<\delta_{1} \zeta_{2}$. Thus, Lemma 4 (ii) and (iii) provide an upper bound of the second term in the above display

$$
\int_{\left|\tau-t^{*}\right|>\frac{1}{3} y^{-\delta_{0}} \zeta_{2}^{-1}} h_{b}(\tau) d \tau \leq \exp \left(-y^{-\varepsilon_{0}}\right)
$$

for $\varepsilon_{0}$ and $y$ sufficiently small and $y^{-\delta_{0}}<\delta_{1} \zeta_{2}$.

For the first term on the right-hand-side of (75), we bound it in a similar way as in constant variance case. In particular, each summand is bounded by

$$
\sup _{\left|\tau-t^{*}\right| \leq \frac{1}{3} y^{-\delta_{0}} \zeta_{2}^{-1}} P\left(\sup _{t \in B_{i}} f(t)>b \mid f(\tau)=\gamma+\frac{z}{b}\right)
$$

For $y^{-\delta_{0}} \zeta_{2}^{-1}<|t-\tau|<\delta_{1}$ and $\left|\tau-t^{*}\right| \leq \frac{1}{3} y^{-\delta_{0}} \zeta_{2}^{-1}$ we have that $\left|t-t^{*}\right|>\frac{2}{3} y^{-\delta_{0}} \zeta_{2}^{-1}$. Using the expansion $\sigma\left(t^{*}\right)-\sigma(t) \sim \Lambda L_{2}\left(\left|t-t^{*}\right|\right)\left|t-t^{*}\right|^{\alpha_{2}}$, we have that

$$
\frac{\sigma(t)}{\sigma(\tau)} \leq 1-\varepsilon_{0} \frac{L_{2}\left(x_{i} \zeta_{2}^{-1}\right)}{L_{2}\left(\zeta_{2}^{-1}\right)} \frac{\left(\zeta_{2}\left|t_{i}-\tau\right|\right)^{\alpha_{2}}}{b^{2}}, \text { for some small } \varepsilon_{0}>0 \text { and }
$$

From the expression of (32) and the inequality (76), for $t \in B_{i} \cap C_{2} \neq \emptyset$ and $x_{i}=\zeta_{2}\left|t_{i}-\tau\right|$, we have that

$$
\mu_{\tau}(t) \leq b+\kappa \sqrt{\frac{L_{2}\left(x_{i} \zeta_{2}^{-1}\right)}{L_{2}\left(\zeta_{2}^{-1}\right)}} \frac{x_{i}^{\alpha_{2} / 2}}{b}-\varepsilon_{0} \frac{L_{2}\left(x_{i} \zeta_{2}^{-1}\right)}{L_{2}\left(\zeta_{2}^{-1}\right)} \frac{x_{i}^{\alpha_{2}}}{b} \leq b-\frac{\varepsilon_{0}}{2} x_{i}^{\alpha_{2}} \frac{L_{2}\left(x_{i} \zeta_{2}^{-1}\right)}{L_{2}\left(\zeta_{2}^{-1}\right)} b^{-1} .
$$

Furthermore, Lemma 1(i) implies that

$$
\operatorname{Var}\left(f_{0}(t)\right)=C_{0}(t, t) \leq 2 \lambda_{2} \frac{L_{2}\left(x_{i} \zeta_{1}^{-1}\right)}{L_{2}\left(\zeta_{1}^{-1}\right)} x_{i}^{\alpha_{2}} b^{-2} .
$$

Thus, the Borel-TIS inequality suggests that

$$
\sup _{\left|\tau-t^{*}\right| \leq \frac{1}{3} y^{-\delta_{0}} \zeta_{2}^{-1}} P\left(\sup _{t \in B_{i}} f(t)>b \mid f(\tau)=\gamma+\frac{z}{b}\right) \leq \exp \left(-x_{i}^{-\varepsilon_{0}}\right),
$$

for some small constant $\varepsilon_{0}$.

Combining the upper bound for the two term on the right side of (75), and putting together all 
$B_{i}$ 's such that $y^{-\delta_{0}}<x_{i}<\delta_{1}$, we have that

$$
\begin{aligned}
& \int_{T} P\left(\frac{1}{m e s\left(A_{\gamma}\right)}>y^{-d} \zeta_{2}^{d}, \sup _{t \in C_{2}} f(t)>b \mid f(\tau)=\gamma+\frac{z}{b}\right) h_{b}(\tau) \frac{q_{b, \tau}(\gamma+z / b)}{b} d \tau d z \\
\leq & \exp \left(-y^{-\varepsilon_{0}}\right)+\sum_{k=0}^{\infty} \kappa\left(y^{-\delta_{0}}+k\right)^{d-1} \exp \left(-\left(y^{-\delta_{0}}+k\right)^{\varepsilon_{0}}\right) \\
\leq & \exp \left(-y^{-\varepsilon_{0} / 2}\right)
\end{aligned}
$$

for some large constant $\kappa>0$ and possible a different choice of $\varepsilon_{0}$.

Case 3: $|t-\tau| \geq \delta_{1}$ and $y<b^{-\lambda}$. The analysis is completely analogous to the Case 3 on Page 22. The only difference is that the variance function $\sigma^{2}(t)$ is non-constant. Given that $\sigma(t)$ is Hölder continuous, all the calculations remain. Therefore, we omit the details and directly reach the bound that

$$
\int_{T} P\left(\frac{1}{m e s\left(A_{\gamma}\right)}>y^{-d} \zeta^{d}, \sup _{|t-\tau|>\delta_{1}} f(t)>b \mid f(\tau)=\gamma+\frac{z}{b}\right) h_{b}(\tau) \frac{q_{b, \tau}(\gamma+z / b)}{b} d \tau d z \leq \exp \left(-y^{-\varepsilon_{0}}\right)
$$

for all $y<b^{-\lambda}$.

Case 4: $|t-\tau| \geq \delta_{1}, y \geq b^{-\lambda}$. We split the bound (74) into two parts.

$$
\begin{aligned}
& \int_{T} P\left(\frac{1}{m e s\left(A_{\gamma}\right)}>y^{-d} \zeta^{d}, \sup _{|t-\tau|>\delta_{1}} f(t)>b \mid f(\tau)=\gamma+\frac{z}{b}\right) h_{b}(\tau) d \tau \\
\leq & \sup _{\left|\tau-t^{*}\right| \leq \delta_{1} / 3} P\left(\frac{1}{m e s\left(A_{\gamma}\right)}>y^{-d} \zeta^{d}, \sup _{|t-\tau|>\delta_{1}} f(t)>b \mid f(\tau)=\gamma+\frac{z}{b}\right) \\
\quad & \quad \int_{\left|\tau-t^{*}\right|>\delta_{1} / 3} h_{b}(\tau) d \tau .
\end{aligned}
$$

From Lemma 4 (iii), the second term on the right side of last inequality can be bound by $\exp \left(-b^{\varepsilon_{0}}\right)$ for some $\varepsilon_{0}>0$. Note that in Case $4, y>b^{-\lambda}$, so this expression can be further bounded by

$$
\int_{\left|\tau-t^{*}\right|>\delta_{1} / 3} h_{b}(\tau) d \tau \leq \exp \left(-\varepsilon_{0} b^{2}\right) \leq \exp \left(-y^{-\varepsilon_{0} / \lambda}\right) .
$$

Now we consider the first term on the right side of (78). On the set $\left|\tau-t^{*}\right|<\delta_{1} / 3$ and $|t-\tau|>\delta_{1}$, there exists some $\varepsilon_{0}$ such that the conditional mean can be bounded from below by

$$
\mu_{\tau}(t) \leq\left(1-\frac{\varepsilon_{0}}{2}\right) b
$$

This is because from condition A4, $\sigma(\tau) \geq \sigma\left(t^{*}\right)-\Lambda L\left(\delta_{1} / 3\right)\left(\delta_{1} / 3\right)^{\alpha}$, for $\left|\tau-t^{*}\right| \leq 1 / 3 \delta_{1}$; while $\sigma(t) \leq \sigma\left(t^{*}\right)-\Lambda L\left(2 \delta_{1} / 3\right)\left(2 \delta_{1} / 3\right)^{\alpha_{2}}$, for $\left|t-t^{*}\right| \geq 2 \delta_{1} / 3$. As a result, there exists a constant $\varepsilon_{0}>0$ such that $\frac{\sigma(t)}{\sigma(\tau)} \leq 1-\varepsilon_{0}$. In addition, the correlation function also drops.

For the rest of case 4 , we follow the same analysis as that of Case 4 on page 23 and derive an 
upper bound for the first term on the right side of (78).

$$
\begin{aligned}
P\left(\frac{1}{m e s\left(A_{\gamma}\right)}>y^{-d} \zeta^{d}, \sup _{|t-\tau| \geq \delta_{1}} f(t)>b \mid f(\tau)=\gamma+\frac{z}{b}\right) & \leq P\left(\sup _{|t-\tau| \geq \delta_{1}} f(t)>b \mid f(\tau)=\gamma+\frac{z}{b}\right) \\
& \leq P\left(\sup _{|t-\tau| \geq \delta_{1}} f_{0}(t)+\mu_{\tau}(t)>b\right) \\
& \leq P\left(\sup _{|t-\tau| \geq \delta_{1}} f_{0}(t)>\varepsilon_{0} b / 2\right) \\
& \leq \exp \left(-\frac{\varepsilon_{0}^{2}}{8 \sigma_{T}^{2}} b^{2}\right) \\
& \leq \exp \left(-y^{-\varepsilon_{0}^{\prime}}\right) .
\end{aligned}
$$

for some $\varepsilon_{0}, \varepsilon_{0}^{\prime}>0$. Combining our result for the first and second term of (78), and for $C_{i}=C_{3}$ for $y \geq b^{-\lambda}$

$$
\text { (174) } \leq \exp \left(-y^{-\varepsilon_{0}}\right), \quad \text { for some possibly smaller } \varepsilon_{0}>0 \text {. }
$$

Summary of the analysis for $I_{1}$. Putting all the results in Cases 1-4 together, we have that there exists a $y_{0}>0$ such that

$$
Q\left(\frac{1}{\operatorname{mes}\left(A_{\gamma}\right)}>y^{-d} \zeta_{2}^{-1}, M>b\right) \leq \exp \left(-y^{-\varepsilon_{0}}\right)
$$

for $0<y<y_{0}$. Thus, for some $\kappa>0$, we have

$$
I_{1}=E^{Q}\left(\frac{1}{m e s\left(A_{\gamma}\right)^{2}} ; M>b\right) \leq\left(\kappa+y_{0}^{-d}\right) \zeta_{2}^{2 d}
$$

The $I_{2}$ term. We are going to derive a lower bound for $I_{2}$ by showing that Lemma 2 and Lemma 3 are valid. Following the same calculation for (78), we reach the result of Lemma 2 (on page 24) that

$$
Q\left(\sup _{|t-\tau| \geq \delta^{\prime}} f(t) \geq \gamma\right) \leq Q\left(\left|t^{*}-\tau\right| \geq \delta^{\prime} / 3\right)+Q\left(\sup _{|t-\tau| \geq \delta^{\prime}} f(t) \geq \gamma,\left|t^{*}-\tau\right|<\delta^{\prime} / 3\right)
$$

The first term on the right-hand-side is controlled by Lemma 4 (iii). The second term can be bounded by a similar analysis as in (80). Thus, we have that

$$
Q\left(\sup _{|t-\tau| \geq \delta^{\prime}} f(t) \geq \gamma\right) \leq e^{-\varepsilon_{0} b^{2}}
$$

for some $\varepsilon_{0}$ small.

Now, we proceed to proving a similar result as in Lemma 3 (page 24). Note that for $x \zeta_{2}{ }^{-1}<\delta^{\prime}$

$$
\begin{aligned}
Q\left(\sup _{x \zeta_{2}^{-1} \leq|t-\tau| \leq \delta^{\prime}} f(t) \geq \gamma\right) \leq Q\left(\sup _{x \zeta_{2}^{-1} \leq|t-\tau| \leq \delta^{\prime}} f(t) \geq \gamma,\left|\tau-t^{*}\right|<x \zeta_{2}^{-1} / 3\right) \\
+Q\left(\left|\tau-t^{*}\right|>x \zeta_{2}^{-1} / 3\right) .
\end{aligned}
$$

Thanks to Lemma 4, the second term on the right-hand-side is bounded by $e^{-x^{\varepsilon_{0}}}$. For the first term, we follow a similar analysis as in Lemma 3. In particular, we can establish a bound for the 
conditional mean $\mu_{\tau}(t)=E(f(\tau+t) \mid \tau, z)$ in the following form

$$
\mu_{\tau}(t) \leq \gamma+\frac{z}{b}-\varepsilon_{0} \frac{x^{\alpha_{2}}}{b}
$$

for all $x \zeta_{2}^{-1}<|t|<\delta^{\prime}$ and $\left|\tau-t^{*}\right|<x \zeta_{2}^{-1} / 3$. With this bound, we follow exactly the same analysis as in Lemma 3 and obtain that

$$
Q\left(\sup _{x \zeta_{2}^{-1} \leq|t-\tau| \leq \delta^{\prime}} f(t) \geq \gamma\right) \leq e^{-x^{\alpha_{2} / 4}}
$$

and thus a similar result in Lemma 3 has been proved. With these results, we use the same analysis as that in (53) and obtain that for some $x$ sufficiently large

$$
I_{2} \geq \varepsilon_{0} x^{-d} \zeta_{2}^{d}
$$

Combining our upper bound for $I_{1}$ and lower bound for $I_{2}$, we conclude the proof for scenario 1.

$$
\sup _{b} \frac{E^{Q} Z_{b}^{2}}{P^{2}(M>b)}=\sup _{b} \frac{I_{1}}{I_{2}^{2}}<\infty
$$

A.2 Proof for scenario 2: $\alpha_{1}<\alpha_{2}$, or $\alpha_{1}=\alpha_{2}$ and $\lim _{x \rightarrow 0} \frac{L_{1}(x)}{L_{2}(x)}=\infty$

In scenario 2, we first consider the covariance function $C(s, t)=\operatorname{cov}(f(s), f(t))$. It satisfies the following conditions:

B1 There exists $\beta_{0} \geq 0, \beta_{1}>0$, such that $\beta_{0}+\beta_{1} \geq \alpha_{1}$, and

$$
\left|C\left(\tau, t+s_{1}\right)-C\left(\tau, t+s_{2}\right)\right| \leq \kappa \max \left(L_{2}\left(\left|s_{1}\right|\right)\left|s_{1}\right|^{\beta_{0}}, L_{2}\left(\left|s_{2}\right|\right)\left|s_{2}\right|^{\beta_{0}}\right)\left|s_{1}-s_{2}\right|^{\beta_{1}}
$$

B2 As $|t-s| \rightarrow 0$,

$$
C(s, s)-C(s, t) \sim \sigma(s)^{2} \Delta_{s} L_{1}(|s-t|)|s-t|^{\alpha_{1}}
$$

B3 There exists $\varepsilon^{\prime \prime}, \delta^{\prime \prime}>0$ such that for $\left|s-t^{*}\right|<\delta^{\prime \prime},|t-s|>2 \delta^{\prime \prime}$, we have

$$
C(s, s)-C(s, t)>\varepsilon^{\prime \prime} .
$$

Therefore, we can basically replicate the analysis in Section 5 for the constant mean by replacing the correlation function $r(s, t)$ with the covariance function $C(s, t)$ and all the derivations are exactly the same except for one place. In the analysis of Case 4 (Page 23), for which we need to provide a bound for

$$
Q\left(\operatorname{mes}\left(A_{\gamma}\right)^{-1}>y^{-d} \zeta_{1}^{d}, \sup _{|t-\tau|>\delta_{1}} f(t)>b\right) .
$$

For this part, we need to following the analysis of Case 4 for scenario 1 (page 43). Other analyses are all the same and therefore are omitted.

\section{B Proof of Lemmas}

Throughout the proof, we used several properties of slowly varying function, which are stated in the next Lemma. 
Lemma 5 Suppose $L(x), x>0$ is a positive continuous slowly varying function, then it has the following properties.

(i) $\forall \beta>0, \exists \delta_{\beta}>0, \kappa_{s}$, s.t. for $\zeta$ satisfying $\zeta^{-1}<\delta_{\beta}, x \leq 1$ we have

$$
\frac{L\left(x \zeta^{-1}\right)}{L\left(\zeta^{-1}\right)} x^{\beta} \leq \kappa_{s}
$$

(ii) $\forall \beta>0, \exists \delta_{\beta}>0, \kappa_{s}>0$, s.t. for $\zeta$ satisfying $\zeta^{-1} x<\delta_{\beta}, x \geq 1$, we have

$$
\frac{L\left(\zeta^{-1} x\right) x^{\beta}}{L\left(\zeta^{-1}\right)} \geq \kappa_{s}^{-1}
$$

This lemma is a direct application of Theorem 1.5.3, and Theorem 1.5.4 in [9].

Proof of Lemma 2, For $|t-\tau| \geq \delta$, according to condition A3, there exits $\varepsilon>0$, such that $r(t, \tau)<1-\varepsilon$. For $b$ large enough, and $0<z<\frac{\varepsilon}{4} b^{2}$, we have

$$
\begin{aligned}
\mu_{\tau}(t) & =\mu(t+\tau)+\frac{r(t+\tau, \tau)}{r(\tau, \tau)}\left(\gamma+\frac{z}{b}-\mu(\tau)\right) \\
& \leq 2 \mu_{T}+(1-\varepsilon)\left(\gamma+\frac{z}{b}\right) \\
& \leq(1-\varepsilon / 2) b
\end{aligned}
$$

and the conditional variance $C_{0}(t, t)=C(t+\tau, t+\tau)-C(t+\tau, \tau)^{2} C(\tau, \tau)^{-1}$ is bounded by $\sigma_{T}^{2}$. Then by the Borel-TIS inequality (Proposition 1), we have that

$$
P\left(\sup _{|t-\tau| \geq \delta} f(t) \geq \gamma \mid f(\tau)=\gamma+\frac{z}{b}\right) \leq e^{-\frac{\varepsilon^{2}}{8 \sigma_{T}^{2}} b^{2}}
$$

Since $z$ is asymptotically exponentially distributed with mean $\sigma(\tau)^{2}$ and $\tau$ is asymptotically uniformly distributed, we have

$$
Q\left(\sup _{|t-\tau|>\delta} f(t) \geq \gamma\right) \leq \sup _{z<\frac{\varepsilon b^{2}}{4}} P\left(\sup _{|t-\tau| \geq \delta} f(t) \geq \gamma \mid f(\tau)=\gamma+\frac{z}{b}\right)+Q\left(z>\varepsilon b^{2} / 4\right) \leq e^{-\varepsilon_{0} b^{2}} .
$$

Proof of Lemma 3. According to conditional Gaussian calculation, we have that

$$
Q\left(b \times(f(\tau)-\gamma) \geq x^{\alpha_{1} / 2}\right) \leq e^{-\varepsilon_{0} x^{\alpha_{1} / 2}} .
$$

Therefore, we only need to consider that $f(\tau)=\gamma+\frac{z}{b}$ for $z<x^{\alpha_{1} / 2}$. Let $\tilde{T}=\left\{t_{1}, \ldots, t_{N}\right\}$ such that:

1. For $i \neq j, i, j \in\{1, \ldots, N\},\left|t_{i}-t_{j}\right|>\zeta_{1}^{-1}$

2. For any $t \in T$, there exists $i \in\{1, \ldots, N\}$, such that $\left|t-t_{i}\right| \leq 2 \zeta_{1}^{-1}$.

Furthermore, let $B_{i}=\left\{t:\left|t-t_{i}\right| \leq 2 \zeta_{1}^{-1}\right\}, i \in\{1,2, \ldots, N\}$. First calculate the upper bound for conditional mean and variance. For $k / \zeta_{1} \leq\left|t_{i}-\tau\right| \leq(k+1) / \zeta_{1}, t \in B_{i}$, and $z<x^{\alpha_{1} / 2}$ according 
to condition A2 and A5, we have that

$$
\begin{aligned}
\mu_{\tau}(t) & \leq b+\frac{z}{b}+\kappa_{\mu} \sqrt{L_{1}(|t|)}|t|^{\alpha_{1} / 2}-\Delta_{\tau} b L_{1}(|t|)|t|^{\alpha_{1}} \\
& \leq b-\frac{\Delta_{\tau}}{2} \frac{L_{1}\left(k \zeta_{1}^{-1}\right)}{L_{1}\left(\zeta_{1}^{-1}\right)} k^{\alpha_{1}} b^{-1} .
\end{aligned}
$$

For the conditional variance, by Lemma 1(i), when $t \in B_{i}$ and $k$ large enough, we have

$$
\begin{aligned}
C_{0}(t, t) & \leq \lambda_{1} L_{1}\left((k+3) \zeta_{1}^{-1}\right)(k+3)^{\alpha_{1}} \zeta_{1}^{-\alpha_{1}} \\
& \leq 2 \lambda_{1} \frac{L_{1}\left(k \zeta_{1}^{-1}\right)}{L_{1}\left(\zeta_{1}^{-1}\right)} k^{\alpha_{1}} b^{-2}
\end{aligned}
$$

According to Lemma 1 (iii), $E\left(\sup _{\left|t+\tau-t_{i}\right| \leq 2 \zeta_{1}^{-1}} f_{0}(t)\right)=O\left(b^{-1}\right)$ as $b \rightarrow \infty$. So for $k$ large enough, we have

$$
E\left[\sup _{t \in B_{i}} f_{0}(t)\right] \leq \frac{\Delta_{\tau}}{4} \frac{L_{1}\left(k \zeta_{1}^{-1}\right)}{L_{1}\left(\zeta_{1}^{-1}\right)} k^{\alpha_{1}} b^{-1} .
$$

By Proposition 11, (85), (86), and (87), we have

$$
P\left(\sup _{\left|t-t_{i}\right| \leq 2 \zeta_{1}^{-1}} f(t) \geq \gamma \mid f(\tau)=\gamma+\frac{z}{b}\right) \leq \exp \left(-\frac{\Delta_{\tau}^{2} L_{1}\left(k \zeta_{1}^{-1}\right) k^{\alpha_{1}}}{64 L_{1}\left(\zeta_{1}^{-1}\right) \lambda_{1}}\right) \leq \exp \left(-\frac{\Delta_{\tau}^{2} k^{\alpha_{1} / 2}}{64 \lambda_{1}}\right) .
$$

The last inequality of the above display is due to Lemma 5 (ii). Note that

$$
P\left(\sup _{x \zeta_{1}^{-1}<|t-\tau|<\delta} f(t)>\gamma \mid f(\tau)=\gamma+\frac{z}{b}\right) \leq \sum_{x \zeta_{1}^{-1}<\left|t_{i}-\tau\right|<\delta^{\prime}} P\left(\sup _{t \in B_{i}} f(t) \geq \gamma \mid f(\tau)=\gamma+\frac{z}{b}\right) .
$$

According to (88), we further bound the above probability by

$$
\begin{aligned}
\sum_{x \zeta_{1}^{-1}<\left|t_{i}-\tau\right|<\delta} P\left(\sup _{t \in B_{i}} f(t) \geq \gamma \mid f(\tau)=\gamma+\frac{z}{b}\right) & \leq O(1) \sum_{k=\lfloor x\rfloor}^{\delta \zeta_{1}} k^{d-1} \exp \left(-\frac{\Delta_{\tau} k^{\alpha_{1} / 2}}{64 \lambda_{1}}\right) \\
& \leq e^{-x^{\alpha_{1} / 2-\varepsilon_{0}}}
\end{aligned}
$$

for $x$ sufficiently large and $\varepsilon_{0}$ small. We integrate the above bound with respect to $(z, \tau)$ under the measure $Q$ and conclude the proof.

Proof of Lemma 4. The proof of this lemma is based on the fact that $P(f(t)>\gamma)$ has the approximation

$$
P(f(t)>\gamma)=\frac{1}{\sqrt{2 \pi}} \frac{\sigma(t)}{\gamma-\mu(t)} \exp \left(-\frac{\gamma-\mu(t)}{2 \sigma(t)}\right)(1+o(1))
$$

combined with the expansion of $\sigma(t)^{2}$ around $t^{*}$,

$$
\sigma(t)^{2}=\sigma\left(t^{*}\right)^{2}-2 \sigma\left(t^{*}\right) \Lambda L_{2}\left(\left|t-t^{*}\right|\right)\left|t-t^{*}\right|^{\alpha_{2}}(1+o(1)) .
$$


After basic calculation of expansion and integration, we can prove that there exist $\varepsilon_{0}, \kappa>0$, such that for $x>\kappa$, we have

$$
\begin{aligned}
\int_{\left|t-t^{*}\right| \leq \zeta_{2}^{-1}} P(f(t)>\gamma) d t & \geq \frac{1}{\sqrt{2 \pi}} \frac{\sigma\left(t^{*}\right) / 2}{\gamma+\mu_{T}} \zeta_{2}^{-d} \exp \left(-\frac{\left(\gamma-\mu\left(t^{*}\right)\right)^{2}}{2 \sigma\left(t^{*}\right)^{2}}\right) \cdot \varepsilon_{0} \\
\int_{x \zeta_{2}^{-1}<\left|t-t^{*}\right|<\delta} P(f(t)>\gamma) d t & \leq \frac{1}{\sqrt{2 \pi}} \frac{\sigma\left(t^{*}\right)}{\gamma-\mu_{T}} \zeta_{2}^{-d} \exp \left(-\frac{\left(\gamma-\mu\left(t^{*}\right)\right)^{2}}{2 \sigma\left(t^{*}\right)^{2}}\right) \exp \left(-x^{\alpha_{2} / 2}\right) \\
\int_{\left|t-t^{*}\right|>\delta} P(f(t)>\gamma) d t & \leq \frac{1}{\sqrt{2 \pi}} \frac{\sigma\left(t^{*}\right)}{\gamma-\mu_{T}} \exp \left(-\frac{\left(\gamma-\mu\left(t^{*}\right)\right)^{2}}{2 \sigma\left(t^{*}\right)^{2}}\right) \exp \left(-\varepsilon_{0} b^{2}\right)
\end{aligned}
$$

Combining the three inequalities above, and noticing that $h_{b}(t)=\frac{P(f(t)>\gamma)}{\int_{t \in T} P(f(t)>\gamma) d t}$, we have the result in this lemma. 